Canard Explosion Near Non-Liénard Type Slow-Fast Hopf Point Peer-reviewed author version

HUZAK, Renato (2018) Canard Explosion Near Non-Liénard Type Slow-Fast Hopf Point. In: Journal of Dynamics and Differential Equations, 31 (2), p. 683-709..

DOI: $10.1007 / \mathrm{s} 10884-018-9645-3$

Handle: http://hdl.handle.net/1942/25654 


\title{
Canard explosion near non-Liénard type slow-fast Hopf point
}

\author{
Renato Huzak \\ Hasselt University, Campus Diepenbeek, Agoralaan Gebouw D, \\ 3590 Diepenbeek, Belgium
}

\begin{abstract}
In this paper we study birth of canards near a smooth slow-fast Hopf point of non-Liénard center type which plays an important role in slowfast codimension 3 saddle and elliptic bifurcations. We show that the number of limit cycles created in the birth of canards in such a slow-fast non-Liénard case is finite. Our paper is also a natural continuation of [DR09] where slow-fast Hopf points of Liénard type have been studied. We use geometric singular perturbation theory and the family blow-up.
\end{abstract}

\section{Introduction}

The goal of this paper is to finish the study of small amplitude limit cycles in slow-fast codimension 3 saddle and elliptic bifurcations. The slow-fast codimension 3 saddle and elliptic bifurcations have been studied in [HDMD13, HDMD14, Huz16], and our paper is the natural continuation of [Huz16] where, studying the cyclicity of the origin in the slow-fast codimension 3 bifurcations, we encountered the birth of canards in a non-Liénard slow-fast Hopf point of center type. Due to the length of the paper [Huz16], we give a detailed study of this non-Liénard slow-fast Hopf case in a separate paper.

Before we explain general planar slow-fast Hopf points in more detail, we will first use a family blow-up to detect the birth of canards in the slow-fast codimension 3 saddle and elliptic bifurcations that we want to study here. These very degenerate codimension 3 bifurcations are given by (see e.g. [HDMD13]):

$$
\left\{\begin{array}{l}
\dot{x}=y \\
\dot{y}=-x y+\epsilon\left(b_{0}+b_{1} x+b_{2} x^{2} \pm x^{3}+x^{4} \bar{H}(x, \lambda)+y^{2} G(x, y, \lambda)\right),
\end{array}\right.
$$

where $\epsilon \geq 0$ is the singular perturbation parameter $(\epsilon \sim 0), b=\left(b_{0}, b_{1}, b_{2}\right) \sim$ $(0,0,0)$ are regular perturbation parameters, $\lambda \in \Lambda$, with $\Lambda$ a compact subset of euclidean space, and where $G$ and $\bar{H}$ are smooth functions (in this paper "smooth" means $C^{\infty}$-smooth). The cyclicity of the origin $(x, y)=(0,0)$ in the family (1) has been studied in [HDMD13, HDMD14, Huz16] under the following condition on the function $\bar{H}: \bar{H}(0, \lambda) \neq 0$ for all $\lambda \in \Lambda$. This condition "breaks" the symmetry in (1) because the coefficient in front of the quartic term $x^{4}$ in 
(1) is nonzero. (For the sake of generality, we have included parameter $\lambda$ in the slow-fast family (1).)

To study small amplitude limit cycles in (1), the idea in [HDMD13, HDMD14, Huz16] was to reduce the codimension of the system by blowing up (1) at $(x, y, b)=(0,0,0)$. The method of reducing codimension consists of two steps (see also [HDMD13]):

1. First, we blow up the origin in the parameter space $b$ by using the following blow-up formula:

$$
\left(b_{0}, b_{1}, b_{2}\right)=\left(r^{3} B_{0}, r^{2} B_{1}, r B_{2}\right), r \geq 0, B=\left(B_{0}, B_{1}, B_{2}\right) \in \mathbb{S}^{2} .
$$

Instead of studying (1), with $b$ kept in a small neighborhood of the origin in $b$-space, it suffices to study (1), with $r \sim 0$ and $B \in \mathbb{S}^{2}$. Thus, after this rescaling in the parameter space $b$, we deal with only one small parameter $r(B \neq(0,0,0))$. Instead of working with the spherical coordinates, in [HDMD13] we introduced 6 charts (or regions) covering the sphere $\mathbb{S}^{2}$ : the jump regions $\left\{B_{0}= \pm 1\right\}\left(\left(B_{1}, B_{2}\right)\right.$ kept in a large compact set $)$, the slowfast Hopf region $\left\{B_{1}=-1\right\}\left(B_{0} \sim 0\right.$ and $B_{2}$ kept in a large compact set), the saddle region $\left\{B_{1}=+1\right\}\left(B_{0} \sim 0\right.$ and $B_{2}$ kept in a large compact set) and the slow-fast Bogdanov-Takens regions $\left\{B_{2}= \pm 1\right\}\left(\left(B_{0}, B_{1}\right) \sim(0,0)\right)$. Taking these compact sets large enough, we can cover the entire sphere (see also [HDMD13]). As we can see from [HDMD13, HDMD14, Huz16], the slow-fast Hopf region represents the most difficult region to deal with, and in this region we will detect our birth of canards.

2. We blow up $(x, y, r)=(0,0,0)$ by using the following family blow-up:

$$
(x, y, r)=\left(u \bar{x}, u^{2} \bar{y}, u \bar{r}\right), u \geq 0, \bar{r} \geq 0,(\bar{x}, \bar{y}, \bar{r}) \in \mathbb{S}^{2},
$$

Instead of using the spherical coordinates, it is more convenient to work with 5 different charts covering the half-sphere: the family directional chart $\{\bar{r}=1\}$ and the phase-directional charts $\{\bar{x}=1\},\{\bar{x}=-1\},\{\bar{y}=1\}$ and $\{\bar{y}=-1\}$. Now, if we want to study the limit cycles of (1) in a small but fixed neighborhood of the origin $(x, y)=(0,0)$, then it suffices to study the blown-up vector field in each chart. For the purpose of this paper we use only the family directional chart $\{\bar{r}=1\}$.

In the family chart $\{\bar{r}=1\}$ of the blow-up (2), system (1) changes to (after division by $u>0, u \sim 0$ ):

$$
\left\{\begin{aligned}
\dot{\bar{x}}= & \bar{y} \\
\dot{\bar{y}}= & -\bar{x} \bar{y}+\epsilon\left(B_{0}+B_{1} \bar{x}+B_{2} \bar{x}^{2} \pm \bar{x}^{3}+u \bar{x}^{4} \bar{H}(u \bar{x}, \lambda)\right. \\
& \left.+u \bar{y}^{2} G\left(u \bar{x}, u^{2} \bar{y}, \lambda\right)\right) .
\end{aligned}\right.
$$

where $(\bar{x}, \bar{y})$ is kept in a large compact set. Now, it is clear that (3) represents a slow-fast family with codimension $\leq 2$, in each region in the parameter space $B$. Thus, we have reduced the codimension of our original system. In the slow-fast Hopf region, (3) has the following form:

$$
\left\{\begin{aligned}
\dot{\bar{x}}= & \bar{y} \\
\dot{\bar{y}}= & -\bar{x} \bar{y}+\epsilon^{2}\left(\epsilon B_{0}-\bar{x}+B_{2} \bar{x}^{2} \pm \bar{x}^{3}+u \bar{x}^{4} \bar{H}(u \bar{x}, \lambda)\right. \\
& \left.+u \bar{y}^{2} G\left(u \bar{x}, u^{2} \bar{y}, \lambda\right)\right)
\end{aligned}\right.
$$


where $u \sim 0$, the parameter $B_{2}$ is kept in a large compact set and where we replace $\left(\epsilon, B_{0}\right)$ by $\left(\epsilon^{2}, \epsilon B_{0}\right)$, with $B_{0} \sim 0$. It is well known (see [HDMD13] or [Huz16]) that limit cycles can occur only in the $\left(\epsilon^{2}, \epsilon B_{0}\right)$-parameter region, with $\epsilon \sim 0$ and $B_{0} \sim 0$. As we will see later in this section, (4) represents a slow-fast Hopf point at $(\bar{x}, \bar{y})=(0,0)$. The slow-fast Hopf point gives rise to a birth of canard limit cycles that occur between large canard limit cycles in the $(\bar{x}, \bar{y})$-space and small limit cycles near $(\bar{x}, \bar{y})=(0,0)$ the size of which tends to 0 as $\epsilon \rightarrow 0$ (we detect the small limit cycles after a family blow-up at $(\bar{x}, \bar{y}, \epsilon)=(0,0,0)$, see Section 2). We denote by $L_{0}$ the limit periodic set near which such a birth of canards occurs, and the goal of our paper is to prove Theorem 3.16 which gives the structure of the difference map defined near $L_{0}$. Then the finite cyclicity of $L_{0}$ (see Theorem 2.1) will follow from Theorem 3.16. Let us recall that in [Huz16] we use Theorem 3.16 to glue together local cyclicity results and to obtain the global cyclicity 2 of $(x, y)=(0,0)$ in $(1)$, when $\bar{H}(0, \lambda)$ is nonzero. For a precise definition of $L_{0}$, see Section 2 .

Let us now explain how system (4) fits into a general framework for planar slow-fast Hopf points. We use the following smooth normal form for a slow-fast Hopf point (see e.g. [DMDR11]):

$$
\left\{\begin{array}{l}
\dot{\bar{x}}=\bar{y} \\
\dot{\bar{y}}=-\bar{x} \bar{y}+\epsilon\left(b_{0}-\bar{x}+O\left(\bar{x}^{2}\right)\right)+O\left(\epsilon \bar{y}^{2}\right),
\end{array}\right.
$$

where $\epsilon$ is the singular parameter, $b_{0} \sim 0$ is a breaking parameter and $O\left(\bar{x}^{2}\right)$ and $O\left(\epsilon \bar{y}^{2}\right)$ are smooth functions. $\left(O\left(\bar{x}^{2}\right)\right.$ does not depend on $\bar{y}$.) It is well known that limit cycles can be created by a (slow-fast) Hopf bifurcation in (5) as we vary the breaking parameter $b_{0}$ (see e.g. [Ben81, DR96, KS01, DR09]). Like in (4), we can study a birth of canards in (5) creating limit cycles that are smaller than detectable canard limit cycles of $(5)$ in the $(\bar{x}, \bar{y})$-space, but larger than small limit cycles of $(5)$ near $(\bar{x}, \bar{y})=(0,0)$ the size of which goes to 0 as $\epsilon \rightarrow 0$. The birth of canards in codimension 1 slow-fast Hopf bifurcations (i.e. (5) with a nonzero coefficient in front of the quadratic term $\bar{x}^{2}$ in $O\left(\bar{x}^{2}\right)$ ) has been studied in [KS01], generalizing the Van der Pol system [DR96]. The paper [DR09] deals with the birth of canards in a Liénard version of (5) (i.e. $O\left(\epsilon \bar{y}^{2}\right) \equiv 0$ in (5)), with a general $O\left(\bar{x}^{2}\right)$-term (see Theorem 5.11 of [DR09]). As far as we know, the birth of canards in the non-Liénard case (i.e. $\left.O\left(\epsilon \bar{y}^{2}\right) \not \equiv 0\right)$ has not yet been studied, and the idea of our paper is to study the special nonLiénard case (4). When $\left(B_{0}, B_{2}, u\right)=(0,0,0),(4)$ is of center type (see Section 2 ) and we obtain Theorem 2.1(ii). When $B_{2} \neq 0$, we deal with the well known codimension 1 slow-fast Hopf point (see Theorem 2.1(i)). Like in Theorem 5.11 of [DR09], in Theorem 2.1 we obtain un upper bound for the cyclicity of $L_{0}$ that is one unit higher that the cyclicity of $L_{0}$. This has been proved in [Huz16] by gluing together different local cyclicity results and by using Theorem 3.16 (see also [DR09]).

Although we deal with the special smooth center case (4), we believe that our methods can be used for a smooth non-Liénard system (5) with a general $O\left(\bar{x}^{2}\right)$-term (i.e. a general center or any finite codimension). This is a topic of further study. When (5) is analytic, we can remove the quadratic term $O\left(\epsilon \bar{y}^{2}\right)$ (see [Huz17] for more details) and we can directly use the result of [DR09].

In Section 2 we define the limit periodic set $L_{0}$ and state our main results. By generalizing the methods introduced in [DR09], in Section 3, we proof our main 
results (Theorem 3.16 and Theorem 2.1). We use symmetries from (4) and from the family blow-up at $(\bar{x}, \bar{y}, \epsilon)=(0,0,0)$ (see Section 2 ), we use smooth normal forms from [DR10] to study transition maps near semi-hyperbolic singularities on the blow-up locus, etc. Before applying results from [DR10], first we need to construct a smooth and symmetric center manifold near the semi-hyperbolic singularities and to find the most suitable structure of such a center manifold.

Our proof (see Section 3) follows similar steps as the one in [DR09] in the Liénard case and consists essentially of two steps. First we find the form of the transition maps near semi-hyperbolic singularities on the blow-up locus (see Theorem 3.8) from which it follows that the non-Liénard term $\epsilon^{2} u \bar{y}^{2} G$ in (4) (i.e the term $u \delta^{3}\left(1-\frac{1}{2} v^{2}\right)^{2} G$ in (14)) turns out to be not relevant in comparison with the dominant (Liénard) term $\epsilon^{2} u \bar{x}^{4} \bar{H}$ in (4) (i.e the term $u \delta^{3} v^{4} \bar{H}$ in (14)) if $\bar{H}(0, \lambda) \neq 0$. In other words, the goal of Section 3.1 is to study the system (14) near the semi-hyperbolic singularities and to show that the Liénard part of (4) produces a principal part of (14). Since the rest of the proof (see Sections $3.2-3.5)$ is very similar to the proof in [DR09] and more technical, we omit here the details. (We refer to [Huz13] for complete proofs.)

\section{Definition of the polycycle $L_{0}$ and statement of results}

For the sake of better readability, in this section we use the same notation as in [Huz16]. We define a segment $\mathcal{B}=\left[-B_{3}^{0}, B_{3}^{0}\right]$, where $B_{3}^{0}>1$ is an arbitrarily large but fixed real number, and consider the following smooth slow-fast family:

$$
Z_{\epsilon, B_{0}, B_{2}, B_{3}, u, \lambda}:\left\{\begin{aligned}
\dot{\bar{x}}= & \bar{y}-\frac{1}{2} \bar{x}^{2} \\
\dot{\bar{y}}= & \epsilon^{2}\left(\epsilon B_{0}-\bar{x}+B_{2} \bar{x}^{2}+B_{3} \bar{x}^{3}+u \bar{x}^{4} \bar{H}(u \bar{x}, \lambda)\right. \\
& \left.+u\left(\bar{y}-\frac{1}{2} \bar{x}^{2}\right)^{2} G\left(u \bar{x}, u^{2}\left(\bar{y}-\frac{1}{2} \bar{x}^{2}\right), \lambda\right)\right),
\end{aligned}\right.
$$

where $\epsilon \geq 0$ is the singular perturbation parameter, $B_{0} \sim 0, B_{2}$ is kept in a compact subset of $\mathbb{R}, B_{3} \in \mathcal{B}, u \sim 0, \lambda \in \Lambda$ and $\bar{H}$ and $G$ are smooth functions. System (6) represents (4) in the Liénard plane $\left\{\bar{y} \rightarrow \bar{y}-\frac{1}{2} \bar{x}^{2}\right\}$ which is more convenient to use the results from [DR10]. For the sake of generality, we add a parameter $B_{3}$.

In this paper we distinguish between two cases:

1. $\left(\mathbf{B}_{\mathbf{2}} \neq \mathbf{0}\right)$ In this case we call $(6)$ slow-fast Hopf bifurcations of codimension 1 and prove that the cyclicity of the polycycle $L_{0}$ is bounded by 2 (see Theorem 2.1(i)).

2. $\left(\mathbf{B}_{\mathbf{2}} \sim \mathbf{0}\right.$ and $\overline{\mathbf{H}}(\mathbf{0}, \lambda) \neq \mathbf{0}$ for all $\left.\lambda \in \boldsymbol{\Lambda}\right)$ This case is referred to as slowfast Hopf bifurcations of center type because (6) has a center at the origin $(\bar{x}, \bar{y})=(0,0)$ when $\left(B_{0}, B_{2}, u\right)=(0,0,0)$. Since $\bar{H}(0, \lambda)$ is nonzero, the cyclicity of $L_{0}$ is bounded by 3 (see Theorem 2.1(ii)).

As we will see in later sections, the cyclicity of $L_{0}$ does not depend on the coefficient $B_{3} \in \mathcal{B}$ because the cubic term $B_{3} \bar{x}^{3}$ in (6) is not a symmetry breaking term. When $B_{3}=+1$ (resp. $B_{3}=-1$ ), we deal with the saddle case (resp. the elliptic case). 
Before we define $L_{0}$ by using a family blow-up at the origin $(\bar{x}, \bar{y}, \epsilon)=(0,0,0)$ in (6), let us observe that the slow-fast family (6) is invariant under

$$
\mathcal{S}:\left(\bar{x}, \bar{y}, \epsilon, B_{0}, B_{2}, B_{3}, u, \lambda, t\right) \rightarrow\left(-\bar{x}, \bar{y}, \epsilon,-B_{0},-B_{2}, B_{3},-u, \lambda,-t\right) .
$$

The symmetry $\mathcal{S}$ plays a crucial role in our paper (see Section 3 ).

\subsection{The family blow-up at the origin in the $(\overline{\mathbf{x}}, \overline{\mathbf{y}}, \epsilon)$-space}

We consider a $\tau:=\left(B_{0}, B_{2}, B_{3}, u, \lambda\right)$-family of vector fields on $\mathbb{R}^{3}$

$$
Z_{\tau}:=Z_{\epsilon, B_{0}, B_{2}, B_{3}, u, \lambda}+0 \frac{\partial}{\partial \epsilon},
$$

where we add the equation $\dot{\epsilon}=0$ to (6), and we define the following singular change of coordinates (i.e. a family blow-up at $(\bar{x}, \bar{y}, \epsilon)=(0,0,0))$ :

$$
\Theta_{1}: \mathbb{R}^{+} \times \mathbb{S}^{2} \rightarrow \mathbb{R}^{3}:(\delta,(\widetilde{x}, \widetilde{y}, w)) \mapsto(\bar{x}, \bar{y}, \epsilon)=\left(\delta \widetilde{x}, \delta^{2} \widetilde{y}, \delta w\right), w \geq 0 .
$$

The blown-up vector field is the pullback of $Z_{\tau}$ divided by $\delta$ :

$$
\bar{Z}_{\tau}:=\frac{1}{\delta} \Theta_{1}^{*} Z_{\tau}
$$

Instead of studying the original vector field $Z_{\tau}$ near $(\bar{x}, \bar{y}, \epsilon)=(0,0,0)$, we study (8) near the blow-up locus $\{0\} \times \mathbb{S}_{+}^{2}$ in different charts.

\subsubsection{Family directional chart $\{\mathrm{w}=1\}$}

In the family chart $\{w=1\}$ we deal with the following family rescaling of $(6)$ :

$$
(\bar{x}, \bar{y})=\left(\epsilon \widetilde{x}, \epsilon^{2} \widetilde{y}\right)
$$

where $(\widetilde{x}, \widetilde{y})$ is kept in an arbitrarily large disk in $\mathbb{R}^{2}$ and $\epsilon \geq 0$. The blown-up field (8) becomes an $(\epsilon, \tau)$-family of 2-dimensional vector fields (we treat $\epsilon$ as a parameter)

$$
Z_{\epsilon, \tau}^{(1)}:\left\{\begin{aligned}
\dot{\tilde{x}}= & \widetilde{y}-\frac{1}{2} \widetilde{x}^{2} \\
\dot{\widetilde{y}}= & B_{0}-\widetilde{x}+B_{2} \epsilon \widetilde{x}^{2}+B_{3} \epsilon^{2} \widetilde{x}^{3}+u \epsilon^{3} \widetilde{x}^{4} \bar{H}(u \epsilon \widetilde{x}, \lambda) \\
& +u \epsilon^{3}\left(\widetilde{y}-\frac{1}{2} \widetilde{x}^{2}\right)^{2} G\left(u \epsilon \widetilde{x}, u^{2} \epsilon^{2}\left(\widetilde{y}-\frac{1}{2} \widetilde{x}^{2}\right), \lambda\right) .
\end{aligned}\right.
$$

In the rest of this section we give some basic properties of (10) that, on the one hand, help us detect the limit periodic set $L_{0}$ (see Section 2.1.3). The properties, on the other hand, will be used for the study of the difference map near $L_{0}$ visible in the family directional charts (see e.g. Sections 3.1 .5 and 3.2). From (7) and (9) it follows that $Z_{\epsilon, \tau}^{(1)}$ is invariant under the symmetry:

$$
\mathcal{S}_{1}:\left(\widetilde{x}, \widetilde{y}, B_{0}, B_{2}, B_{3}, u, \epsilon, \lambda, t\right) \rightarrow\left(-\widetilde{x}, \widetilde{y},-B_{0},-B_{2}, B_{3},-u, \epsilon, \lambda,-t\right) .
$$

When $B_{0}=\epsilon=0, Z_{\epsilon, \tau}^{(1)}$ has the following form:

$$
\left\{\begin{array}{l}
\dot{\widetilde{x}}=\widetilde{y}-\frac{1}{2} \widetilde{x}^{2} \\
\dot{\tilde{y}}=-\widetilde{x}
\end{array}\right.
$$

Since the vector field (12) is invariant under $(\widetilde{x}, \widetilde{y}, t) \rightarrow(-\widetilde{x}, \widetilde{y},-t),(12)$ has a center at the origin $(\widetilde{x}, \widetilde{y})=(0,0)$. Moreover, $(12)$ is the dual of the differential 
1-form $\omega_{0}=\widetilde{x} d \widetilde{x}+\left(\widetilde{y}-\frac{1}{2} \widetilde{x}^{2}\right) d \widetilde{y}$, and the function $H(\widetilde{x}, \widetilde{y})=e^{-\widetilde{y}}\left(\widetilde{y}-\frac{1}{2} \widetilde{x}^{2}+1\right)$ represents a first integral if we use the function $-e^{-\widetilde{y}}$ as integrating factor (i.e. $\left.-e^{-\widetilde{y}} w_{0}=d H\right)$. Clearly, $\{H(\widetilde{x}, \widetilde{y})=1\}$ represents the center $(\widetilde{x}, \widetilde{y})=(0,0)$, denoted by $L_{1}$ in Figure 1 , and $\{H(\widetilde{x}, \widetilde{y})=0\}$ represents the orbit $\gamma$ in Figure 1 which separates the closed level curves of $H$, for $h \in] 0,1$ [, from the open level curves, parametrized by $h<0$.

\subsubsection{Phase-directional chart $\{\widetilde{\mathbf{y}}=1\}$}

To study the end points of $\gamma$, we use the following blow-up formula in the phase-directional chart $\{\widetilde{y}=1\}$ :

$$
(\bar{x}, \bar{y}, \epsilon)=\left(\delta v, \delta^{2}, \delta w\right) .
$$

The blown-up field (8) becomes:

$$
Z_{\tau}^{(2)}:\left\{\begin{array}{l}
\dot{v}=1-\frac{1}{2} v^{2}+\frac{1}{2} w^{2} v D(\delta, w, v, \tau) \\
\dot{\delta}=-\frac{1}{2} \delta w^{2} D(\delta, w, v, \tau) \\
\dot{w}=\frac{1}{2} w^{3} D(\delta, w, v, \tau)
\end{array}\right.
$$

where $D(\delta, w, v, \tau)=-\left(w B_{0}-v+B_{2} \delta v^{2}+B_{3} \delta^{2} v^{3}+u \delta^{3} v^{4} \bar{H}(u \delta v, \lambda)+u \delta^{3}(1-\right.$ $\left.\left.\frac{1}{2} v^{2}\right)^{2} G\left(u \delta v, u^{2} \delta^{2}\left(1-\frac{1}{2} v^{2}\right), \lambda\right)\right)$. From (13) and the symmetry $\mathcal{S}$ it follows that $Z_{\tau}^{(2)}$ is invariant under the following symmetries:

$$
\begin{aligned}
& \mathcal{S}_{2}:\left(v, \delta, w, B_{0}, B_{2}, B_{3}, u, \lambda, t\right) \rightarrow\left(v,-\delta,-w,-B_{0},-B_{2}, B_{3},-u, \lambda, t\right), \\
& \mathcal{S}_{3}:\left(v, \delta, w, B_{0}, B_{2}, B_{3}, u, \lambda, t\right) \rightarrow\left(-v, \delta, w,-B_{0},-B_{2}, B_{3},-u, \lambda,-t\right) .
\end{aligned}
$$

The blow-up formula (13) also implies that $\bar{Z}_{\tau}$ is invariant under the symmetry:

$$
\mathcal{S}_{4}:(v, \delta, w, \tau, t) \rightarrow(-v,-\delta,-w, \tau,-t) .
$$

When $\delta=w=0$, (14) has two singularities, $v= \pm \sqrt{2}$. The eigenvalues at $(v, \delta, w)=(\sqrt{2}, 0,0)=: S_{2}$ are given by $(-\sqrt{2}, 0,0)$ and at $(v, \delta, w)=$ $(-\sqrt{2}, 0,0)=: S_{1}$ by $(\sqrt{2}, 0,0)$. This implies that $(14)$ has at $S_{2}$ (resp. $\left.S_{1}\right)$ a semi-hyperbolic singularity with a two-dimensional center manifold and the $v$-axis as stable manifold (resp. unstable manifold). The points $S_{1,2}$ are the end points of $\gamma$ and of the critical curve $\{v= \pm \sqrt{2}, \delta \geq 0, w=0\}$ in the blown-up space.

When we study $L_{0}$, we don't need to use the other phase-directional charts $(\{\widetilde{x}= \pm 1\}$ and $\{\widetilde{y}=-1\})$. See Section 2.1.3.

\subsubsection{Combining the family chart and the phase-directional chart $\{\widetilde{\mathbf{y}}=\mathbf{1}\}$}

In this section we detect the limit periodic set $L_{0}$, at level $B_{0}=0$, using Sections 2.1.1 and 2.1.2. The blown-up vector field $\bar{Z}_{\tau}$ is a $\tau$-family of 3 -dimensional vector fields defined in a small neighborhood of the blow-up locus, denoted by $\bar{S}$ in Figure 1. For $\epsilon=0$, this family depends on the parameter $B_{0} \sim 0$ (see (10) and (14)). The vector field (12) represents $\bar{Z}_{\tau}$ on $\bar{S}$, when $B_{0}=0$. Clearly, the $\alpha$-limit set of $\gamma$ is $S_{2}$ and the $\omega$-limit set of $\gamma$ is $S_{1}$, and the limit periodic set $L_{0}$ is defined as the union of $\gamma$ and the (regular) arc $C$ between $S_{1}$ and $S_{2}$. See Figure 1. 


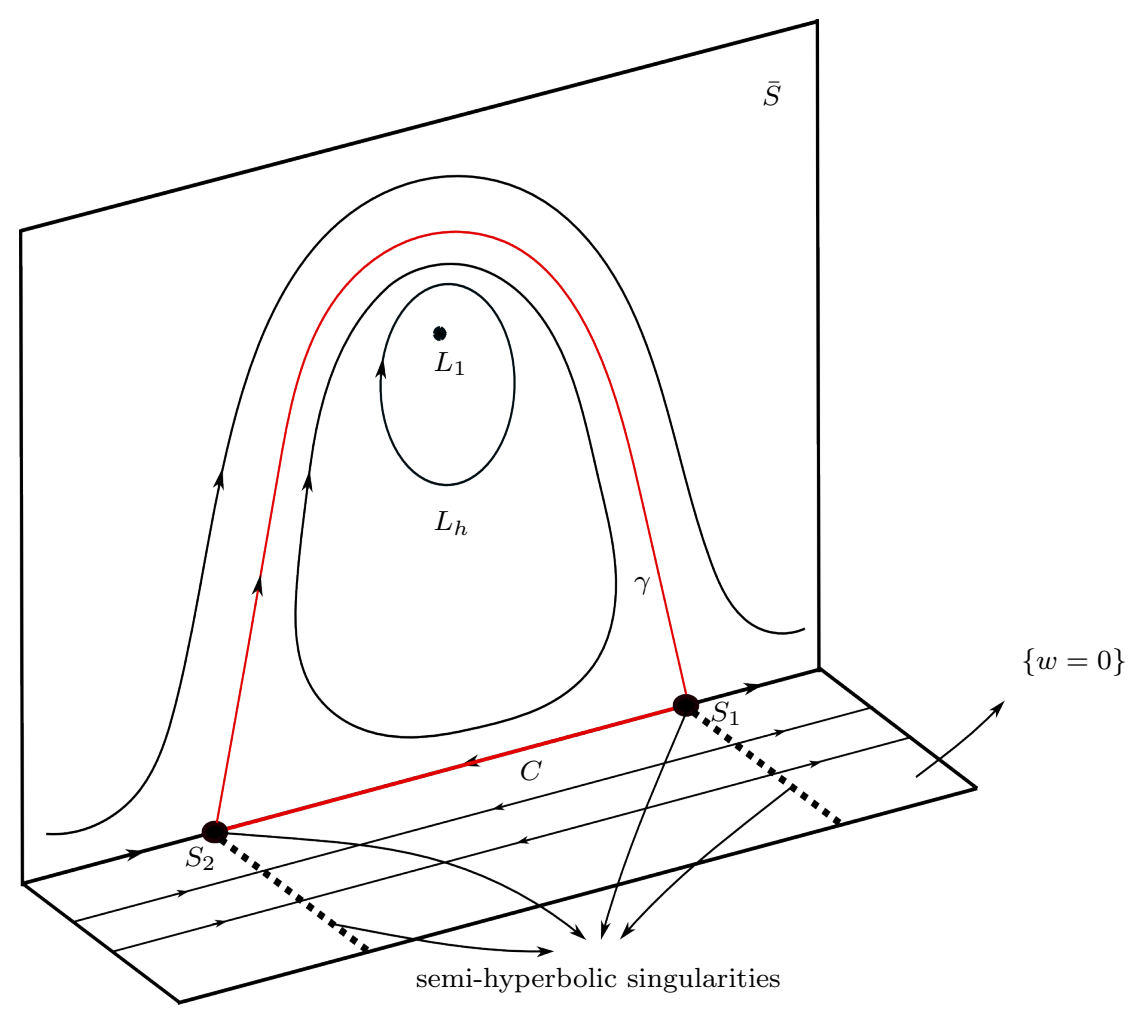

Figure 1: The dynamics of the vector field $\bar{Z}_{\tau}$ for $\epsilon=B_{0}=0$ and the singular cycle $L_{0}=\gamma \cup C$.

\subsection{Difference map and statement of results}

Our goal is to study limit cycles Hausdorff close to $L_{0}$ as zeros of a difference map. First, we choose sections $\Sigma_{ \pm}$transverse to $C$ and sections $T_{ \pm}$transverse to $\gamma$ (see Figure 2). The chosen sections are parametrized by two regular parameters (see Section 3 for precise definitions of $\Sigma_{ \pm}$and $T_{ \pm}$). Now we define transition maps near $L_{0}$ (see Figure 2):

(a) the regular transition map $\mathcal{F}_{\tau}$ near $C$ from $\Sigma_{-}$to $\Sigma_{+}$, defined by the flow of $Z_{\tau}^{(2)}$,

(b) the Dulac transition maps $\mathcal{D}_{\tau}^{ \pm}$from $\Sigma_{ \pm}$to $T_{ \pm}$, defined by the flow of $\pm Z_{\tau}^{(2)}$

(c) the regular transition map $\mathcal{G}_{\tau}$ near $\gamma$ from $T_{-}$to $T_{+}$, defined by the flow of $-Z_{\epsilon, \tau}^{(1)}$,

and the difference map

$$
\Omega_{\tau}\left(\delta^{\prime}, w^{\prime}\right)=\mathcal{D}_{\tau}^{+} \circ \mathcal{F}_{\tau}\left(\delta^{\prime}, w^{\prime}\right)-\mathcal{G}_{\tau} \circ \mathcal{D}_{\tau}^{-}\left(\delta^{\prime}, w^{\prime}\right),
$$

where $\left(\delta^{\prime}, w^{\prime}\right)$ are the regular parameters on $\Sigma_{-}$. The intersection $C \cap \Sigma_{-}$is given by $\left(\delta^{\prime}, w^{\prime}\right)=(0,0)$. 
To define the cyclicity of $L_{0}$, it is convenient to parametrize $\Sigma_{-}$by $\left(\delta^{\prime}, w^{\prime}\right)=$ $(\delta, w) \sim(0,0)$, where $(\delta, w)$ are the coordinates of $Z_{\tau}^{(2)}$, and $T_{+}$by $(h, \epsilon)$, where $h \sim 0$ is the value of the Hamiltonian $H$ (see Section 2.1.1). Then $\Omega_{\tau}(\delta, w)=$ $\left(w_{\tau}(\delta, w), 0\right)$, where $w_{\tau}$ represents the $h$-component of the difference map $\Omega_{\tau}$ (see also Section 3 ). We say that the cyclicity of $L_{0}$ at $B_{2}=\bar{B}_{2}$ is bounded by $M \in \mathbb{N}$ if there exist $\epsilon_{0}>0, d>0$ and a small neighborhood $\mathcal{V}$ of $\left(0, \bar{B}_{2}, 0\right)$ in $\left(B_{0}, B_{2}, u\right)$-space such that for each $\left.\left.\epsilon \in\right] 0, \epsilon_{0}\right],\left(B_{0}, B_{2}, u\right) \in \mathcal{V}, B_{3} \in \mathcal{B}, \lambda \in \Lambda$ and $\left(B_{0}, B_{2}, u\right) \neq(0,0,0)$ the number of zeros (counting multiplicity) of $w_{\tau}(\delta, w)$ on $s_{\epsilon}=\{(\delta, w) ; \delta w=\epsilon,(\delta, w) \in B(d), \delta \geq 0, w \geq 0\}$ is bounded by $M$. $(B(d)$ is a ball of radius $d$ at $(\delta, w)=(0,0)$.) The smallest $M$ with this property is called the cyclicity of $L_{0}$ at $B_{2}=\bar{B}_{2}$. See also Section 3.4.

Theorem 2.1. The following statements are true:

(i) Suppose that $\bar{B}_{2} \neq 0$. Then the cyclicity of $L_{0}$ at $B_{2}=\bar{B}_{2}$ is bounded by 2.

(ii) If $\bar{H}(0, \lambda) \neq 0$ for all $\lambda \in \Lambda$, then the cyclicity of $L_{0}$ at $B_{2}=0$ is bounded by 3 .

Theorem 2.1 will be proved in Section 3. The proof of Theorem 2.1 consists of two steps. First, we have to prove Theorem 3.16 which gives the structure of the difference map $w_{\tau}(\delta, w)$. (Let us recall that Theorem 3.16 plays an important role in [Huz16].) Then we study the number of zeros of $w_{\tau}(\delta, w)$ by using the so-called Lie-derivative of $w_{\tau}(\delta, w)$ along the vector field $\mathcal{Y}=\delta \frac{\partial}{\partial \delta}-w \frac{\partial}{\partial w}$ and Rolle's theorem (see Sections 3.4 and 3.5).

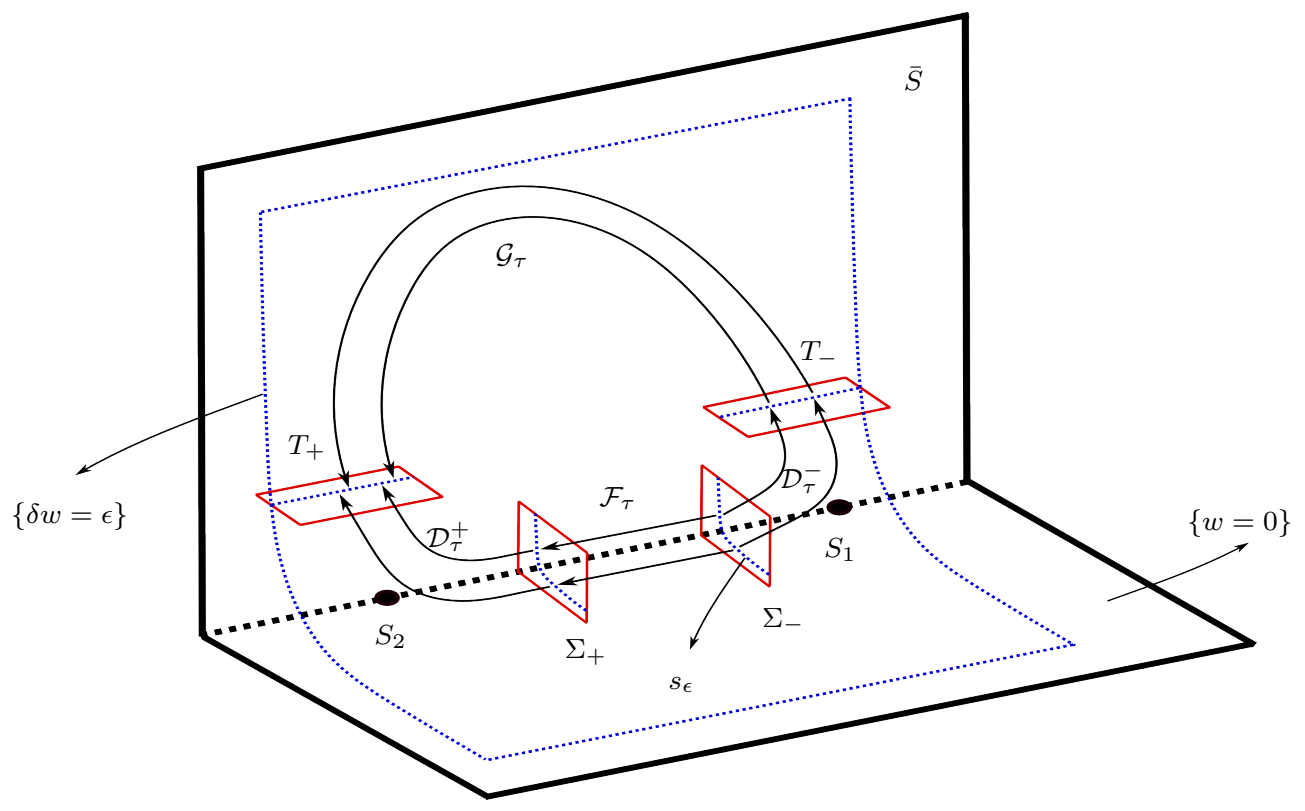

Figure 2: The maps $\mathcal{D}_{\tau}^{ \pm}, \mathcal{F}_{\tau}, \mathcal{G}_{\tau}$. 


\section{Proof of Theorem 2.1}

In order to prove Theorem 3.16, we study the form of the (global) transition maps $\mathcal{F}_{\tau}$ and $\mathcal{G}_{\tau}$, and the (local) Dulac transition maps $\mathcal{D}_{\tau}^{ \pm}$. We start with $\mathcal{D}_{\tau}^{ \pm}$.

We will often denote by $O\left(r_{1}, \ldots, r_{j}\right)$ a function $\sum_{i=1}^{j} R_{i} r_{i}$, where $R_{i}$ and $r_{i}$ are functions of $x$. If all these functions are smooth in $x$, then we say that the function $O\left(r_{1}, \ldots, r_{j}\right)$ is smooth in $x$.

\subsection{Transitions near $S_{1}$ and $S_{2}$}

To study the blown-up field $\bar{Z}_{\tau}$ around $S_{1,2}$, we will use the vector field (14). The symmetry (17) implies that the study of (14) in backward time near $S_{1}$ in the region $\{\delta \geq 0, w \geq 0, v<0\}$ follows from a study of (14) in forward time near $S_{2}$ in the region $\{\delta \leq 0, w \leq 0, v>0\}$. Thus, it suffices to study the point $S_{2}$. The results for $S_{1}$ will be obtained by using the symmetry $\mathcal{S}_{4}$ (see Section 3.1.3). Instead of working with $Z_{\tau}^{(2)}$ near $S_{2}$, it is more convenient to use the following equivalent field near $S_{2}$ :

$$
\widetilde{Z}_{\tau}:=D(\delta, w, v, \tau)^{-1} Z_{\tau}^{(2)},
$$

where $D(\delta, w, v, \tau)$ is defined in (14). The vector field $\widetilde{Z}_{\tau}$ is well defined in a small and fixed neighborhood of the point $S_{2}$ because the parameter $\tau$ is bounded.

To find the structure of the transition map $\mathcal{D}_{\tau}^{+}$, we have to linearize the hyperbolic $v$-component in (19). The linearization method consists of 3 steps. First, we prove existence of a smooth and $\mathcal{S}_{2}$-invariant center manifold at $S_{2}$ and study the structure of such a center manifold (see Section 3.1.1). Then we straighten the center manifold and study the structure of the linear part of the hyperbolic component in (19) (see Section 3.1.2). Finally, in Section 3.1.4 we linearize the vector field (41), obtained in Section 3.1.2. In Section 3.1.5 we find the form of $\mathcal{D}_{\tau}^{+}$where $T_{+}$is parametrized by $(h, \epsilon)$ (see Theorem 3.11).

\subsubsection{A smooth and symmetric center manifold}

We saw in Section 2.1 that $S_{2}$ is a semi-hyperbolic singular point of $Z_{\tau}^{(2)}$ (or $\widetilde{Z}_{\tau}$ ), for any value of the parameter $\tau$. A center manifold $C_{\tau}$ at $S_{2}$ can be represented by $\left\{v=\sqrt{2}+v_{0}(\delta, w, \tau)\right\}$, where $v_{0}(\delta, 0, \tau) \equiv 0$. Following the well known theory [DR96], we can suppose that $C_{\tau}$ is a smooth family, i.e. the function $v_{0}(\delta, w, \tau)$ is $C^{\infty}$. In the rest of this section, we restrict ourselves to smooth $\tau$-families of center manifolds $C_{\tau}$ near $S_{2}$.

In this section, we show that it is possible to fix a smooth $\mathcal{S}_{2}$-invariant choice $v_{0}(\delta, w, \tau)$, and we find that $v_{0}(\delta, w, \tau)-v_{0}(0, w, \tau)=w^{2} O\left(B_{2} \delta, B_{3} \delta^{2}, u \delta^{3}, \delta^{4}\right)$. As we will see in later sections, this plays an important role. We begin with the intersection $C_{\tau} \cap\{\delta=0\}$.

Lemma 3.1. The restriction of any (smooth) center manifold $C_{\tau}$ of $\widetilde{Z}_{\tau}$ at $S_{2}$ to $\{\delta=0\}$ does not depend on $\left(B_{2}, B_{3}, u, \lambda\right)$ and on the choice of $C_{\tau}$ and it is the graph of a smooth function $v=\sqrt{2}+\bar{v}_{0}\left(w, B_{0}\right)$ where

$$
\bar{v}_{0}\left(w, B_{0}\right)=v_{0}(0, w, \tau)=w^{2}\left(\frac{1}{\sqrt{2}}-\frac{1}{2} B_{0} w\right)+O\left(w^{4}\right) .
$$


We have that $\bar{v}_{0}\left(w, B_{0}\right)=\bar{v}_{0}\left(-w,-B_{0}\right)$.

Proof. See [Huz13].

Let us recall that $\tau=\left(B_{0}, B_{2}, B_{3}, u, \lambda\right)$. We denote by $\tau^{*}$ the parameter $\left(-B_{0},-B_{2}, B_{3},-u, \lambda\right)$.

Proposition 3.2. We can choose $v_{0}(\delta, w, \tau)$ such that

$$
v_{0}(\delta, w, \tau)=v_{0}\left(-\delta,-w, \tau^{*}\right) .
$$

Proof. Let $C_{\tau}=\left\{v=\sqrt{2}+v_{0}(\delta, w, \tau)\right\}$ be a center manifold of $\widetilde{Z}_{\tau}$ at $S_{2}$. Since system $\widetilde{Z}_{\tau}$ is invariant under $\mathcal{S}_{2}$ defined in $(15),\left\{v=\sqrt{2}+v_{0}\left(-\delta,-w, \tau^{*}\right)\right\}$ is also a center manifold of $\widetilde{Z}_{\tau}$.

The center manifold $C_{\tau}$ satisfies

$$
\begin{aligned}
& \frac{1-\frac{1}{2}\left(\sqrt{2}+v_{0}(\delta, w, \tau)\right)^{2}}{D\left(\delta, w, \sqrt{2}+v_{0}(\delta, w, \tau), \tau\right)} \\
& \quad+\frac{1}{2} w^{2}\left(\sqrt{2}+v_{0}(\delta, w, \tau)+\delta \frac{\partial v_{0}}{\partial \delta}(\delta, w, \tau)-w \frac{\partial v_{0}}{\partial w}(\delta, w, \tau)\right)=0 .
\end{aligned}
$$

We define the $n$-jet of $v_{0}(\delta, w, \tau)$ at $w=0$, for each $n \in \mathbb{N} \cup\{\infty\}$ :

$$
j^{n} v_{0}(\delta, w, \tau)=\sum_{k=0}^{n} v_{0}^{k}(\delta, \tau) w^{k} .
$$

The formal series of $v_{0}(\delta, w, \tau)$ in powers of $w$ at $w=0$ does not depend on the chosen center manifold $C_{\tau}$. Indeed, using $(22)$ it can be easily seen that $v_{0}^{0}(\delta, \tau)=v_{0}^{1}(\delta, \tau) \equiv 0$ and $v_{0}^{k}(\delta, \tau), k \geq 2$, is solution of an explicit solvable function. In other words,

$$
j^{\infty} v_{0}(\delta, w, \tau)=j^{\infty} v_{0}\left(-\delta,-w, \tau^{*}\right) .
$$

Hence the infinite jet of $v_{0}$ satisfies the $\mathcal{S}_{2}$-symmetry. We define now

$$
V(\delta, w, \tau)=\left(\sqrt{2}+v_{0}(\delta, w, \tau)\right) \chi_{\{w \geq 0\}}+\left(\sqrt{2}+v_{0}\left(-\delta,-w, \tau^{*}\right)\right) \chi_{\{w<0\}},
$$

where $\chi_{A}$ is the characteristic function of the set $A$. Hence we consider the restriction of $C_{\tau}$ to $\{w \geq 0\}$ and extension of it in the set $\{w \leq 0\}$ by $\mathcal{S}_{2^{-}}$ symmetry. It is clear that $V$ is smooth for $w \neq 0$. It remains to prove that $V$ is smooth along $\{w=0\}$. To prove this, we compare the right-hand partial derivatives of any order at $w=0$ and the corresponding left-hand partial derivatives at $w=0$. From the definition of $V$ and the property (23) follows that $V$ is smooth along $\{w=0\}$. Note that $\{v=V(\delta, w, \tau)\}$ is a center manifold of $\widetilde{Z}_{\tau}$ and $\mathcal{S}_{2}$-invariant by the definition of $V$. This completes the proof.

From now on we suppose that $v_{0}$ is smooth and invariant under the symmetry $\mathcal{S}_{2}$. To study the structure of $v_{0}$, we need the following lemma:

Lemma 3.3. Let $\Sigma=w \frac{\partial}{\partial w}+\alpha \delta \frac{\partial}{\partial \delta}$ for some $\alpha \neq 0$. Let $f(\delta, w, \tau)$ be a smooth function which verifies:

$$
(1+O(w, \delta)) f(\delta, w, \tau)+A w^{2}(1+O(w, \delta)) \Sigma f(\delta, w, \tau)=O\left(\delta^{k}\right)
$$

for some $k \in \mathbb{N}_{1}$, a constant $A>0$ and smooth functions $O(w, \delta), O\left(\delta^{k}\right)$. Then $f(\delta, w, \tau)=O\left(\delta^{k}\right)$. 
Proof. See [DR09], Lemma 4.5.

Proposition 3.4. We can find smooth functions $\Psi_{k}(\delta, w, \tau), k=1,2,3,4$, such that:

$$
v_{0}(\delta, w, \tau)=\bar{v}_{0}\left(w, B_{0}\right)+w^{2}\left(B_{2} \delta \Psi_{1}+B_{3} \delta^{2} \Psi_{2}+u \delta^{3} \Psi_{3}+\delta^{4} \Psi_{4}\right),
$$

where $\Psi_{1}(0,0, \tau)=-1, \Psi_{2}(0,0, \tau)=-\sqrt{2}, \Psi_{3}(0,0, \tau)=-2 \bar{H}(0, \lambda), \Psi_{4}(0,0, \tau)=$ 0 . Moreover, we can suppose that $\Psi_{1}$ and $\Psi_{2}$ do not depend on the parameter $u$.

Proof. We can write:

$$
v_{0}(\delta, w, \tau)=\bar{v}_{0}\left(w, B_{0}\right)+V(\delta, w, \tau),
$$

where $V=O(\delta)$. Putting $v_{0}=\bar{v}_{0}+V$ in (22), we get

$$
\begin{aligned}
-\sqrt{2} \bar{v}_{0}- & \frac{1}{2} \bar{v}_{0}^{2}-\sqrt{2} V-\bar{v}_{0} V-\frac{1}{2} V^{2} \\
& +\frac{1}{2} w^{2}\left(\sqrt{2}+\bar{v}_{0}+V-\Sigma \bar{v}_{0}-\Sigma V\right) D\left(\delta, w, \sqrt{2}+\bar{v}_{0}+V, \tau\right)=0
\end{aligned}
$$

where $\Sigma:=w \frac{\partial}{\partial w}-\delta \frac{\partial}{\partial \delta}$. Taking into account the definition of $\bar{v}_{0}$ and (27) we obtain:

$$
\begin{aligned}
-\sqrt{2} V- & \bar{v}_{0} V-\frac{1}{2} V^{2}-\frac{1}{2} w^{2}\left(\sqrt{2}+\bar{v}_{0}-\Sigma \bar{v}_{0}\right) D\left(0, w, \sqrt{2}+\bar{v}_{0}, \tau\right) \\
& +\frac{1}{2} w^{2}\left(\sqrt{2}+\bar{v}_{0}+V-\Sigma \bar{v}_{0}-\Sigma V\right) D\left(\delta, w, \sqrt{2}+\bar{v}_{0}+V, \tau\right)=0,
\end{aligned}
$$

or equivalently,

$$
\begin{aligned}
-\sqrt{2} V-\bar{v}_{0} V-\frac{1}{2} V^{2}+\frac{1}{2} w^{2}(V-\Sigma V) D\left(\delta, w, \sqrt{2}+\bar{v}_{0}+V, \tau\right) \\
+\frac{1}{2} w^{2}\left(\sqrt{2}+\bar{v}_{0}-\Sigma \bar{v}_{0}\right)\left(D\left(\delta, w, \sqrt{2}+\bar{v}_{0}+V, \tau\right)-D\left(0, w, \sqrt{2}+\bar{v}_{0}, \tau\right)\right)=0 .
\end{aligned}
$$

Bearing in mind that $V=O(\delta), \bar{v}_{0}=O\left(w^{2}\right)$ and $\Sigma \bar{v}_{0}=O\left(w^{2}\right)$, (28) changes to

$$
\begin{aligned}
& V\left(-\sqrt{2}+O\left(\delta, w^{2}\right)\right)-\frac{1}{2} w^{2}(\sqrt{2}+O(\delta, w)) \Sigma V \\
& =-\frac{1}{2} w^{2}\left(\sqrt{2}+O\left(w^{2}\right)\right)\left(D\left(\delta, w, \sqrt{2}+\bar{v}_{0}+V, \tau\right)-D\left(0, w, \sqrt{2}+\bar{v}_{0}, \tau\right)\right) .
\end{aligned}
$$

Let us write $\eta=\sqrt{2}+\bar{v}_{0}+V$. We have

$$
\begin{aligned}
D(\delta, w, \eta, \tau) & -D\left(0, w, \sqrt{2}+\bar{v}_{0}, \tau\right) \\
= & -\left(-V+B_{2} \delta \eta^{2}+B_{3} \delta^{2} \eta^{3}+u \delta^{3} \eta^{4} \bar{H}(u \delta \eta, \lambda)\right. \\
& \left.+u \delta^{3}\left(1-\frac{1}{2} \eta^{2}\right)^{2} G\left(u \delta \eta, u^{2} \delta^{2}\left(1-\frac{1}{2} \eta^{2}\right), \lambda\right)\right) .
\end{aligned}
$$

Using the above expression and (29) we finally get

$$
\begin{aligned}
V+ & \frac{1}{2} w^{2}(1+O(\delta, w)) \Sigma V \\
= & -\frac{1}{2} w^{2}(1+O(\delta, w))\left(B_{2} \delta \eta^{2}+B_{3} \delta^{2} \eta^{3}+u \delta^{3} \eta^{4} \bar{H}(u \delta \eta, \lambda)\right. \\
& \left.+u \delta^{3}\left(1-\frac{1}{2} \eta^{2}\right)^{2} G\left(u \delta \eta, u^{2} \delta^{2}\left(1-\frac{1}{2} \eta^{2}\right), \lambda\right)\right) .
\end{aligned}
$$


From (30) it follows that $V=w^{2} \bar{V}$, for some smooth function $\bar{V}$, and

$$
\begin{aligned}
(1+O(\delta, w)) \bar{V}+\frac{1}{2} w^{2}(1+O(\delta, w)) \Sigma \bar{V} \\
=-\frac{1}{2}\left(B_{2} \delta \eta^{2}+B_{3} \delta^{2} \eta^{3}+u \delta^{3} \eta^{4} \bar{H}(u \delta \eta, \lambda)\right. \\
\left.\quad+u \delta^{3}\left(1-\frac{1}{2} \eta^{2}\right)^{2} G\left(u \delta \eta, u^{2} \delta^{2}\left(1-\frac{1}{2} \eta^{2}\right), \lambda\right)\right),
\end{aligned}
$$

for some new functions $O(\delta, w)$. If we denote the right hand side of (31) by $R$, then there exist smooth functions $R_{3}, \ldots, R_{6}$ such that:

$R=\delta R_{3}$,

$R_{3}=\delta R_{4}-B_{2}(1+O(\delta, w))$

$R_{4}=\delta R_{5}-B_{3} \sqrt{2}(1+O(\delta, w))$,

$R_{5}=\delta R_{6}+u(-2 \bar{H}(0, \lambda)+O(\delta, w))$ and

$R_{6}=u^{2}\left(-2 \sqrt{2}\left(\partial_{1} \bar{H}\right)(0, \lambda)+O(\delta, w)\right)$

where we use the fact that $1-\frac{1}{2} \eta^{2}=O\left(w^{2}\right)$.

Let us denote the left hand side of $(31)$ by $A(\bar{V})$. The symbol $A$ is any operator $A(f)=(1+O(\delta, w)) f+\frac{1}{2} w^{2}(1+O(\delta, w)) \Sigma f$. We can prove that $A(\delta f)=\delta A(f)$, up to changing $O(\delta, w)$ in the expression of $A(f)$.

We rely on Lemma 3.3. Since $A(\bar{V})=\delta R_{3}$, Lemma 3.3 implies that there exists a smooth function $\bar{V}_{3}$ such that $\bar{V}=\delta \bar{V}_{3}$. We have that $\delta R_{3}=A(\bar{V})=$ $A\left(\delta \bar{V}_{3}\right)=\delta A\left(\bar{V}_{3}\right)$. As a consequence, $A\left(\bar{V}_{3}\right)=R_{3}=\delta R_{4}-B_{2}(1+O(\delta, w))$. Then we have that $\bar{V}_{3}(0,0, \tau)=-B_{2}$ and, by Lemma 3.3, $\bar{V}_{3}=\delta \bar{V}_{4}+O_{1}\left(B_{2}\right)$ where $\bar{V}_{4}, O_{1}\left(B_{2}\right)$ are smooth.

Working modulo $B_{2}$, it implies that $\delta R_{4}=A\left(\bar{V}_{3}\right)=\delta A\left(\bar{V}_{4}\right)$. Hence $A\left(\bar{V}_{4}\right)=$ $R_{4}=\delta R_{5}-B_{3} \sqrt{2}(1+O(\delta, w))$ modulo $B_{2}$. As a consequence, we have that $\bar{V}_{4}(0,0, \tau)=-B_{3} \sqrt{2}+O\left(B_{2}\right)$ and, by Lemma $3.3, \bar{V}_{4}=\delta \bar{V}_{5}+O_{2}\left(B_{2}, B_{3}\right)$ where $\bar{V}_{5}, \mathrm{O}_{2}\left(B_{2}, B_{3}\right)$ are smooth.

Working modulo $B_{2}$ and $B_{3}$, it implies that $\delta R_{5}=A\left(\bar{V}_{4}\right)=\delta A\left(\bar{V}_{5}\right)$. Hence $A\left(\bar{V}_{5}\right)=R_{5}=\delta R_{6}+u(-2 \bar{H}(0, \lambda)+O(\delta, w))$ modulo $B_{2}$ and $B_{3}$. As a consequence, we have that $\bar{V}_{5}(0,0, \tau)=-2 u \bar{H}(0, \lambda)+O\left(B_{2}\right)+O\left(B_{3}\right)$ and, by Lemma $3.3, \bar{V}_{5}=\delta \bar{V}_{6}+O_{3}\left(B_{2}, B_{3}, u\right)$ where $\bar{V}_{6}, O_{3}\left(B_{2}, B_{3}, u\right)$ are smooth.

Working modulo $B_{2}, B_{3}$ and $u$, it implies that $\delta R_{6}=A\left(\bar{V}_{5}\right)=\delta A\left(\bar{V}_{6}\right)$. Hence $A\left(\bar{V}_{6}\right)=R_{6}=u^{2}\left(-2 \sqrt{2}\left(\partial_{1} \bar{H}\right)(0, \lambda)+O(\delta, w)\right)$ modulo $B_{2}, B_{3}$ and $u$. As a consequence, we have that $\bar{V}_{6}(0,0, \tau)=O\left(B_{2}, B_{3}, u\right)$.

We have proved that $V=w^{2} \bar{V}=w^{2} \delta \bar{V}_{3}=w^{2} \delta\left(\delta\left(\delta\left(\delta \bar{V}_{6}+O_{3}\left(B_{2}, B_{3}, u\right)\right)+\right.\right.$ $\left.\left.\mathrm{O}_{2}\left(B_{2}, B_{3}\right)\right)+O_{1}\left(B_{2}\right)\right)$. After regrouping terms this gives

$$
\begin{array}{r}
V=w^{2}\left(B_{2} \delta \Psi_{1}(\delta, w, \tau)+B_{3} \delta^{2} \Psi_{2}(\delta, w, \tau)\right. \\
\left.+u \delta^{3} \Psi_{3}(\delta, w, \tau)+\delta^{4} \Psi_{4}(\delta, w, \tau)\right),
\end{array}
$$

where $\Phi_{k}$ are smooth, $\Phi_{1}(0,0, \tau)=-1, \Phi_{2}(0,0, \tau)=-\sqrt{2}, \Phi_{3}(0,0, \tau)=$ $-2 \bar{H}(0, \lambda)$ and $\Phi_{4}(0,0, \tau)=0$.

To see that $\Psi_{1}$ and $\Psi_{2}$ can be taken to be independent of $u$, let us write

$$
v_{0}=\widetilde{v}_{0}+\widetilde{V},
$$

where $\widetilde{v}_{0}=v_{0}\left(\delta, w, B_{0}, B_{2}, B_{3}, 0, \lambda\right)$ and $\widetilde{V}=O(u)$. As in the case $v_{0}=\bar{v}_{0}+V$ we put $\widetilde{v}_{0}\left(v_{0}\right.$ for $\left.u=0\right)$ and $\widetilde{v}_{0}+\widetilde{V}$ in (22) and we obtain

$$
\widetilde{V}+\frac{1}{2} w^{2}(1+O(\delta, w)) \Sigma \widetilde{V}=w^{2} u \delta^{3}(-2 \bar{H}(0, \lambda)+O(\delta, w))
$$


As a consequence of Lemma 3.3, we have that $\widetilde{V}=w^{2} u \delta^{3}(-2 \bar{H}(0, \lambda)+O(\delta, w))$, for some new $O(\delta, w)$. Now we can get rid of the $u$-dependent terms in $\Psi_{1}$ and $\Psi_{2}$ by putting them in $u \delta^{3} \Psi_{3}(\delta, w, \tau)$. This completes the proof.

\subsubsection{Straightening of the center manifold}

We continue working at the point $S_{2}$. We define the following smooth $\tau$-family of diffeomorphisms (in a small neighborhood of $S_{2}$ ):

$$
(v, \delta, w) \rightarrow(z, \delta, w), z=v-\left(\sqrt{2}+v_{0}(\delta, w, \tau)\right),
$$

where $v_{0}$ is fixed in Section 3.1.1. The fixed center manifold is given now by $C_{\tau}=\{z=0\}$ and the equation $\widetilde{Z}_{\tau}$ has the following form

$$
\left\{\begin{array}{l}
\dot{z}=\frac{1-\frac{1}{2}\left(\sqrt{2}+v_{0}+z\right)^{2}}{D\left(\delta, w, \sqrt{2}+v_{0}+z, \tau\right)}+\frac{1}{2} w^{2}\left(\sqrt{2}+v_{0}+z+\delta \frac{\partial v_{0}}{\partial \delta}-w \frac{\partial v_{0}}{\partial w}\right) \\
\dot{\delta}=-\frac{1}{2} \delta w^{2} \\
\dot{w}=\frac{1}{2} w^{3} .
\end{array}\right.
$$

Taking into account (22) the $z$-component in (33) changes to

$$
\dot{z}=\left(\frac{1-\frac{1}{2}\left(\sqrt{2}+v_{0}+z\right)^{2}}{D\left(\delta, w, \sqrt{2}+v_{0}+z, \tau\right)}-\frac{1-\frac{1}{2}\left(\sqrt{2}+v_{0}\right)^{2}}{D\left(\delta, w, \sqrt{2}+v_{0}, \tau\right)}\right)+\frac{1}{2} w^{2} z .
$$

Proposition 3.5. There exist smooth functions $A\left(w, B_{0}\right), R(z, \delta, w, \tau), \phi_{k}(\delta, w, \tau)$, for $k=1,2,3,4$, such that the z-component (34) can be written as

$$
\dot{z}=-\left(A+B_{2} \delta \phi_{1}+B_{3} \delta^{2} \phi_{2}+u \delta^{3} \phi_{3}+\delta^{4} \phi_{4}+z R\right) z,
$$

where $\phi_{1}(0,0, \tau)=\sqrt{2}, \phi_{2}(0,0, \tau)=2, \phi_{3}(0,0, \tau)=2 \sqrt{2} \bar{H}(0, \lambda), \phi_{4}(0,0, \tau)=$ $0, A\left(w, B_{0}\right)=\frac{1-\frac{1}{2}\left(\sqrt{2}+\bar{v}_{0}\right)\left(2 w B_{0}-\sqrt{2}-\bar{v}_{0}\right)}{\left(w B_{0}-\sqrt{2}-\bar{v}_{0}\right)^{2}}-\frac{1}{2} w^{2}$ and where $R=R_{0}\left(z, w, B_{0}\right)+$ $O\left(B_{2} \delta, B_{3} \delta^{2}, u \delta^{3}, \delta^{4}\right)$.

Proof. The function $D(\delta, w, v, \tau)$ is given by:

$D(\delta, w, v, \tau)=-\left(w B_{0}-v+B_{2} \delta v^{2}+B_{3} \delta^{2} v^{3}+u \delta^{3} v^{4} \bar{H}(u \delta v, \lambda)+u \delta^{3}(1-\right.$ $\left.\left.\frac{1}{2} v^{2}\right)^{2} G\left(u \delta v, u^{2} \delta^{2}\left(1-\frac{1}{2} v^{2}\right), \lambda\right)\right)$. It is clear that

$$
\frac{1-\frac{1}{2}\left(\sqrt{2}+v_{0}+z\right)^{2}}{-\left(w B_{0}-\sqrt{2}-v_{0}-z+\alpha\right)}=\frac{1-\frac{1}{2}\left(\sqrt{2}+v_{0}+z\right)^{2}}{-\left(w B_{0}-\sqrt{2}-v_{0}-z\right)}+\alpha . L,
$$

where $L(\alpha, z, \delta, w, \tau)$ is smooth and $\alpha \sim 0$. Let us write $\eta_{0}=\sqrt{2}+v_{0}$ and $\eta=\sqrt{2}+v_{0}+z$. We use (36) where

$\alpha=\alpha_{1}:=B_{2} \delta \eta^{2}+B_{3} \delta^{2} \eta^{3}+u \delta^{3} \eta^{4} \bar{H}(u \delta \eta, \lambda)+u \delta^{3}\left(1-\frac{1}{2} \eta^{2}\right)^{2} G\left(u \delta \eta, u^{2} \delta^{2}(1-\right.$ $\left.\left.\frac{1}{2} \eta^{2}\right), \lambda\right)$ and

$\alpha=\alpha_{0}:=B_{2} \delta \eta_{0}^{2}+B_{3} \delta^{2} \eta_{0}^{3}+u \delta^{3} \eta_{0}^{4} \bar{H}\left(u \delta \eta_{0}, \lambda\right)+u \delta^{3}\left(1-\frac{1}{2} \eta_{0}^{2}\right)^{2} G\left(u \delta \eta_{0}, u^{2} \delta^{2}(1-\right.$ $\left.\left.\frac{1}{2} \eta_{0}^{2}\right), \lambda\right)$ :

$$
\begin{aligned}
& \frac{1-\frac{1}{2} \eta^{2}}{D(\delta, w, \eta, \tau)}-\frac{1-\frac{1}{2} \eta_{0}^{2}}{D\left(\delta, w, \eta_{0}, \tau\right)} \\
= & \frac{1-\frac{1}{2} \eta^{2}}{-\left(w B_{0}-\eta\right)}+\alpha_{1} L\left(\alpha_{1}, z, \delta, w, \tau\right)-\frac{1-\frac{1}{2} \eta_{0}^{2}}{-\left(w B_{0}-\eta_{0}\right)}-\alpha_{0} L\left(\alpha_{0}, 0, \delta, w, \tau\right) \\
= & \frac{1-\frac{1}{2} \eta^{2}}{-\left(w B_{0}-\eta\right)}-\frac{1-\frac{1}{2} \eta_{0}^{2}}{-\left(w B_{0}-\eta_{0}\right)}+B_{2} \delta p_{1}+B_{3} \delta^{2} p_{2}+u \delta^{3} p_{3},
\end{aligned}
$$


where $p_{k}(z, \delta, w, \tau)$, for $k=1,2,3$, is a smooth function, and $p_{1}=L\left(\alpha_{1}, z, \delta, w, \tau\right) \eta^{2}-L\left(\alpha_{0}, 0, \delta, w, \tau\right) \eta_{0}^{2}$,

$p_{2}=L\left(\alpha_{1}, z, \delta, w, \tau\right) \eta^{3}-L\left(\alpha_{0}, 0, \delta, w, \tau\right) \eta_{0}^{3}$ and

$p_{3}=L\left(\alpha_{1}, z, \delta, w, \tau\right)\left(\eta^{4} \bar{H}(u \delta \eta, \lambda)+\left(1-\frac{1}{2} \eta^{2}\right)^{2} G\left(u \delta \eta, u^{2} \delta^{2}\left(1-\frac{1}{2} \eta^{2}\right), \lambda\right)\right)$

$-L\left(\alpha_{0}, 0, \delta, w, \tau\right)\left(\eta_{0}^{4} \bar{H}\left(u \delta \eta_{0}, \lambda\right)+\left(1-\frac{1}{2} \eta_{0}^{2}\right)^{2} G\left(u \delta \eta_{0}, u^{2} \delta^{2}\left(1-\frac{1}{2} \eta_{0}^{2}\right), \lambda\right)\right)$.

A direct computation shows that $p_{k}(z, \delta, w, \tau)=z \bar{p}_{k}(z, \delta, w, \tau)$, for $k=1,2,3$, where $\bar{p}_{1}=-\sqrt{2}+O(z, \delta, w), \bar{p}_{2}=-2+O(z, \delta, w)$ and $\bar{p}_{3}=-2 \sqrt{2} \bar{H}(0, \lambda)+$ $O(z, \delta, w)$.

Using (25) for $v_{0}$ we have

$$
\begin{aligned}
& \frac{1-\frac{1}{2} \eta^{2}}{-\left(w B_{0}-\eta\right)}-\frac{1-\frac{1}{2} \eta_{0}^{2}}{-\left(w B_{0}-\eta_{0}\right)}+\frac{1}{2} w^{2} z \\
& =-\left(\frac{1-\frac{1}{2} \eta_{0}\left(2 w B_{0}-\eta_{0}\right)-\frac{1}{2} z\left(w B_{0}-\eta_{0}\right)}{\left(w B_{0}-\eta_{0}\right)\left(w B_{0}-\eta\right)}-\frac{1}{2} w^{2}\right) z \\
& =-\left(p_{0}^{1}+B_{2} \delta p_{1}^{1}+B_{3} \delta^{2} p_{2}^{1}+u \delta^{3} p_{3}^{1}+\delta^{4} p_{4}^{1}\right) z
\end{aligned}
$$

with

$$
p_{0}^{1}\left(z, w, B_{0}\right)=\frac{1-\frac{1}{2}\left(\sqrt{2}+\bar{v}_{0}\right)\left(2 w B_{0}-\sqrt{2}-\bar{v}_{0}\right)-\frac{1}{2} z\left(w B_{0}-\sqrt{2}-\bar{v}_{0}\right)}{\left(w B_{0}-\sqrt{2}-\bar{v}_{0}\right)\left(w B_{0}-\sqrt{2}-\bar{v}_{0}-z\right)}-\frac{1}{2} w^{2}
$$

and, for $\mathrm{k}=1,2,3,4$,

$$
p_{k}^{1}(z, \delta, w, \tau)=w^{2} \Psi_{k}(\delta, w, \tau) M\left(v_{0}(\delta, w, \tau)-\bar{v}_{0}\left(w, B_{0}\right), z, w, B_{0}\right),
$$

where $\Psi_{k}$ are introduced in $(25)$ and $M\left(\beta, z, w, B_{0}\right)$ is a smooth function.

Putting $\widetilde{\phi}_{k}=-\bar{p}_{k}+p_{k}^{1}$, for $k=1,2,3$, and $\widetilde{\phi}_{4}=p_{4}^{1}$, we can rewrite $(34)$ :

$$
\dot{z}=-\left(p_{0}^{1}+B_{2} \delta \widetilde{\phi}_{1}+B_{3} \delta^{2} \widetilde{\phi}_{2}+u \delta^{3} \widetilde{\phi}_{3}+\delta^{4} \widetilde{\phi}_{4}\right) z
$$

Writing $A\left(w, B_{0}\right)=p_{0}^{1}\left(0, w, B_{0}\right)$ and $\phi_{k}(\delta, w, \tau)=\widetilde{\phi}_{k}(0, \delta, w, \tau)$, for $k=1,2,3,4$, (40) becomes (35).

Following Proposition 3.5, (33) can be written as:

$$
\left\{\begin{aligned}
\dot{z}= & -\left(A\left(w, B_{0}\right)+B_{2} \delta \phi_{1}(\delta, w, \tau)\right. \\
& \left.+B_{3} \delta^{2} \phi_{2}(\delta, w, \tau)+u \delta^{3} \phi_{3}(\delta, w, \tau)+\delta^{4} \phi_{4}(\delta, w, \tau)+z R(z, \delta, w, \tau)\right) z \\
\dot{\delta}=- & \frac{1}{2} \delta w^{2} \\
\dot{w}= & \frac{1}{2} w^{3} .
\end{aligned}\right.
$$

The functions $A, \phi_{2}$ and $\phi_{4}$ in (41) have the following symmetry properties:

Proposition 3.6. We have

$$
A\left(-w, B_{0}\right)-A\left(w, B_{0}\right)=A\left(w,-B_{0}\right)-A\left(w, B_{0}\right)=-\sqrt{2} B_{0} w\left(1+O\left(w^{2}\right)\right) .
$$

It is possible to choose $\phi_{2}$ and $\phi_{4}$ in a way that

$$
\begin{aligned}
& \phi_{2}(-\delta,-w, \tau)-\phi_{2}(\delta, w, \tau)=O\left(B_{0} w\right)+O\left(B_{2}\right) \\
& \phi_{4}(-\delta,-w, \tau)-\phi_{4}(\delta, w, \tau)=O\left(B_{0} w\right)+O\left(B_{2}\right)+O(u) .
\end{aligned}
$$


Proof. As $\bar{v}_{0}\left(w, B_{0}\right)=\bar{v}_{0}\left(-w,-B_{0}\right)$, we have $A\left(-w,-B_{0}\right)-A\left(w, B_{0}\right)=0$. It implies that $A\left(-w, B_{0}\right)-A\left(w, B_{0}\right)=O\left(w B_{0}\right)$. Using the fact that $\bar{v}_{0}=$ $O\left(w^{2}\right)$, we find that $\left.\frac{\partial}{\partial w}\left(A\left(-w, B_{0}\right)-A\left(w, B_{0}\right)\right)\right|_{w=0}=-\sqrt{2} B_{0}$. Hence we have $A\left(-w, B_{0}\right)-A\left(w, B_{0}\right)=-\sqrt{2} B_{0} w\left(1+O\left(w^{2}\right)\right)$ due to the fact that $A\left(-w, B_{0}\right)-$ $A\left(w, B_{0}\right)$ is an odd function in variable $w$.

Consider now $\phi_{2}$ and $\phi_{4}$. We will prove that it is possible to regroup terms in (40) in a way that new functions $\widetilde{\phi}_{2}$ and $\widetilde{\phi}_{4}$ in (40) have the following symmetry properties:

$$
\begin{aligned}
& \widetilde{\phi}_{2}(z,-\delta,-w, \tau)-\widetilde{\phi}_{2}(z, \delta, w, \tau)=O\left(B_{0} w\right)+O\left(B_{2}\right) \\
& \widetilde{\phi}_{4}(z,-\delta,-w, \tau)-\widetilde{\phi}_{4}(z, \delta, w, \tau)=O\left(B_{0} w\right)+O\left(B_{2}\right)+O(u) .
\end{aligned}
$$

Then, it suffices to put $z=0$ in the above expressions. Let us recall that $\widetilde{\phi}_{k}=-\bar{p}_{k}+p_{k}^{1}$, for $k=1,2,3$, and $\widetilde{\phi}_{4}=p_{4}^{1}$, where $\bar{p}_{k}$ is defined after (37) and $p_{k}^{1}$ is defined in (39).

Since $v_{0}$ is $\mathcal{S}_{2}$-invariant, it is clear that $L(\alpha, z, \delta, w, \tau)=L\left(\alpha, z,-\delta,-w, \tau^{*}\right)$ where $L$ is defined in (36) and $\tau^{*}=\left(-B_{0},-B_{2}, B_{3},-u, \lambda\right)$. As a consequence, we have $\bar{p}_{2}(z, \delta, w, \tau)=\bar{p}_{2}\left(z,-\delta,-w, \tau^{*}\right)$. Moreover, $\bar{p}_{2}(z, \delta, 0, \tau)$ does not depend on $B_{0}$ due to the fact that $v_{0}=O\left(w^{2}\right)$. Hence, we obtain

$$
\bar{p}_{2}(z,-\delta,-w, \tau)-\bar{p}_{2}(z, \delta, w, \tau)=O\left(B_{0} w\right)+O\left(B_{2}\right)+O(u) .
$$

Based on Proposition 3.4 and (36), we have $\bar{p}_{2}=\left.\bar{p}_{2}\right|_{u=0}+O\left(u \delta^{3}\right)$. Hence we get

$$
\begin{aligned}
B_{2} \delta \bar{p}_{1}+B_{3} \delta^{2} \bar{p}_{2}+u \delta^{3} \bar{p}_{3} & =B_{2} \delta \bar{p}_{1}+\left.B_{3} \delta^{2} \bar{p}_{2}\right|_{u=0}+u \delta^{3}\left(\bar{p}_{3}+O\left(B_{3} \delta^{2}\right)\right) \\
& =: B_{2} \delta \widetilde{p}_{1}+B_{3} \delta^{2} \widetilde{p}_{2}+u \delta^{3} \widetilde{p}_{3} .
\end{aligned}
$$

Since $\widetilde{p}_{2}=\left.\bar{p}_{2}\right|_{u=0}$ does not depend on $u,(43)$ implies that

$$
\widetilde{p}_{2}(z,-\delta,-w, \tau)-\widetilde{p}_{2}(z, \delta, w, \tau)=O\left(B_{0} w\right)+O\left(B_{2}\right) .
$$

We know, by Proposition 3.4, that $\Psi_{2}$ in (39) does not depend on $u$. As a consequence we have that $p_{2}^{1}=\left.p_{2}^{1}\right|_{u=0}+O\left(u \delta^{3}\right)$. Hence we obtain

$$
\begin{aligned}
B_{2} \delta & p_{1}^{1}+B_{3} \delta^{2} p_{2}^{1}+u \delta^{3} p_{3}^{1}+\delta^{4} p_{4}^{1} \\
& =B_{2} \delta p_{1}^{1}+\left.B_{3} \delta^{2} p_{2}^{1}\right|_{u=0}+u \delta^{3}\left(p_{3}^{1}+O\left(B_{3} \delta^{2}\right)\right)+\delta^{4} p_{4}^{1} \\
& =B_{2} \delta p_{1}^{1}+\left.B_{3} \delta^{2} p_{2}^{1}\right|_{u=0}+u \delta^{3}\left(p_{3}^{1}+O\left(B_{3} \delta^{2}, \delta\right)\right)+\left.\delta^{4} p_{4}^{1}\right|_{u=0} \\
& =B_{2} \delta p_{1}^{1}+B_{3} \delta^{2}\left(\left.p_{2}^{1}\right|_{u=0}+O\left(\delta^{2}\right)\right)+u \delta^{3}\left(p_{3}^{1}+O\left(B_{3} \delta^{2}, \delta\right)\right)+\left.\delta^{4} p_{4}^{1}\right|_{u=0, B_{3}=0} \\
& =: B_{2} \delta \widetilde{p}_{1}^{1}+B_{3} \delta^{2} \widetilde{p}_{2}^{1}+u \delta^{3} \widetilde{p}_{3}^{1}+\delta^{4} \widetilde{p}_{4}^{1} .
\end{aligned}
$$

Notice that $\widetilde{p}_{2}^{1}$ is $u$-independent and $\widetilde{p}_{4}^{1}$ is $B_{3}$-independent.

Using the $\mathcal{S}_{2}$-invariance of (38) we have that

$$
\begin{gathered}
B_{3} \delta^{2}\left(\widetilde{p}_{2}^{1}(z,-\delta,-w, \tau)-\widetilde{p}_{2}^{1}(z, \delta, w, \tau)\right) \\
+\delta^{4}\left(\widetilde{p}_{4}^{1}(z,-\delta,-w, \tau)-\widetilde{p}_{4}^{1}(z, \delta, w, \tau)\right)=O\left(w^{2} B_{0}\right)+O\left(w^{2} B_{2}\right)+O\left(w^{2} u\right) .
\end{gathered}
$$

Using (45) and the above mentioned properties of $\widetilde{p}_{2}^{1}$ and $\widetilde{p}_{4}^{1}$, we get

$$
\begin{gathered}
B_{3} \delta^{2}\left(\widetilde{p}_{2}^{1}(z,-\delta,-w, \tau)-\widetilde{p}_{2}^{1}(z, \delta, w, \tau)\right)=O\left(w B_{0}\right)+O\left(B_{2}\right) \\
\delta^{4}\left(\widetilde{p}_{4}^{1}(z,-\delta,-w, \tau)-\widetilde{p}_{4}^{1}(z, \delta, w, \tau)\right)=O\left(w B_{0}\right)+O\left(B_{2}\right)+O(u) .
\end{gathered}
$$


Now, it is clear that

$$
\begin{gathered}
\widetilde{p}_{2}^{1}(z,-\delta,-w, \tau)-\widetilde{p}_{2}^{1}(z, \delta, w, \tau)=O\left(w B_{0}\right)+O\left(B_{2}\right) \\
\widetilde{p}_{4}^{1}(z,-\delta,-w, \tau)-\widetilde{p}_{4}^{1}(z, \delta, w, \tau)=O\left(w B_{0}\right)+O\left(B_{2}\right)+O(u),
\end{gathered}
$$

for some new O-functions.

Define $\widetilde{\phi}_{k}:=-\widetilde{p}_{k}+\widetilde{p}_{k}^{1}$, for $k=1,2,3$, and $\widetilde{\phi}_{4}:=\widetilde{p}_{4}^{1}$. Using the above expressions and (44) we obtain (42).

Let us from now on fix some choice $\left(\phi_{2}, \phi_{4}\right)$ as in Proposition 3.6.

\subsubsection{Study of $Z_{\tau}^{(2)}$ near $S_{1}$}

As the system (14) (i.e. $Z_{\tau}^{(2)}$ ) is invariant under the transformation $\mathcal{S}_{4}$ defined in (17), we can use $\left\{v=-\sqrt{2}-v_{0}(-\delta,-w, \tau)\right\}$ as a smooth symmetric center manifold at the point $S_{1}$ where $\left\{v=\sqrt{2}+v_{0}(\delta, w, \tau)\right\}$ is the (fixed) smooth symmetric center manifold at $S_{2}$. It implies that the study of (14) in backward time near $S_{1}$ in the region $\left\{\delta \geq 0, w \geq 0, v \geq-\sqrt{2}-v_{0}(-\delta,-w, \tau)\right\}$ is equivalent to the study of $\widetilde{Z}_{\tau}$ near $S_{2}$ in the region $\left\{\delta \leq 0, w \leq 0, v \leq \sqrt{2}+v_{0}(\delta, w, \tau)\right\}$. Thus, in the coordinates $(z, \delta, w)$, where $z=v-\sqrt{2}-v_{0}(\delta, w, \tau)$, we focus on (41) on $\{\delta \geq 0, w \geq 0, z \leq 0\}$, to study $\widetilde{Z}_{\tau}$ near $S_{2}$, and we focus on (41) on $\{\delta \leq 0, w \leq 0, z \leq 0\}$, to study $-Z_{\tau}^{(2)}$ near $S_{1}$.

\subsubsection{Normally linearizing the equation (41) along $\{z=0\}$}

As the functions $A+B_{2} \delta \phi_{1}+B_{3} \delta^{2} \phi_{2}+u \delta^{3} \phi_{3}+\delta^{4} \phi_{4}$ and $R(z, \delta, w, \tau)$ in (41) are invariant under $\mathcal{S}_{2}$, defined in (15), and $A\left(0, B_{0}\right)=1>0$, we have the following normal linearization theorem (see [DR10] (Theorem 1.1.) or [DR09] (Theorem 4.8.)):

Theorem 3.7. There exists a smooth family $G_{\tau}(z, \delta, w):(z, \delta, w) \rightarrow(Z, \Delta, W)$ of local diffeomorphisms, defined on a ( $\tau$-uniform) neighborhood of $(z, \delta, w)=$ $(0,0,0)$, which brings the equation (41), for $\widetilde{Z}_{\tau}$ near the point $S_{2}$, into the following normally linearized form:

$$
\left\{\begin{array}{l}
\dot{Z}=-\left(A\left(W, B_{0}\right)+B_{2} \Delta \phi_{1}(\Delta, W, \tau)+B_{3} \Delta^{2} \phi_{2}(\Delta, W, \tau)\right. \\
\dot{\Delta}=-\frac{1}{2} \Delta W^{2} \\
\dot{W}=\frac{1}{2} W^{3} .
\end{array}\right.
$$

The diffeomorphisms $G_{\tau}$ preserve each line $\{\delta w=$ const $\}$; this means that $G_{\tau}(z, \delta, w)=\left(z\left(1+z g_{\tau}(z, \delta, w)\right), \delta, w\right)$ where $g_{\tau}$ is a smooth family of functions. The functions $A, \phi_{k}$ are the ones given in (41) (i.e. A, $\phi_{k}$ are given in Proposition 3.5 taking into account Proposition 3.6). Moreover the diffeomorphisms $G_{\tau}$ commute with the symmetry $\mathcal{S}_{2}$.

Remark 1. We have, by Theorem 3.7, $G_{\tau}^{-1}(Z, \Delta, W)=\left(Z\left(1+\Gamma_{\tau}(Z, \Delta, W)\right)\right.$, $\Delta, W)$ where $\Gamma_{\tau}$ is a smooth family of functions and $\Gamma_{\tau}(Z, \Delta, W)=O(Z)$. The inverses $G_{\tau}^{-1}$ commute with the symmetry $\mathcal{S}_{2}$ because $G_{\tau}$ commute with $\mathcal{S}_{2}$. As a consequence, we have that $\Gamma_{\tau}(Z, \Delta, W)-\Gamma_{\tau}(Z,-\Delta,-W)=O\left(B_{0}\right)+O\left(B_{2}\right)+$ 
$O(u)$. As $B_{2}$ and $u$ are accompanied by, respectively, $\delta$ and $\delta^{3}$ in the expression (40), we get $\Gamma_{\tau}(Z, \Delta, W)-\Gamma_{\tau}(Z,-\Delta,-W)=O\left(B_{0}\right)+O\left(B_{2} \Delta\right)+O\left(u \Delta^{3}\right)($ see also [DR10] and [DR09]).

Section 3.1.3 and Theorem 3.7 imply that for the study of $\widetilde{Z}_{\tau}$ near $S_{2}$ we have to use (46) on $\{\Delta \geq 0, W \geq 0, Z \leq 0\}$, and to study (14) near $S_{1}$ in backward time we can use (46) on $\{\Delta \leq 0, W \leq 0, Z \leq 0\}$. Hence we use (46) on $\{\Delta \geq 0, W \geq 0, Z \leq 0\}$ to study $\widetilde{Z}_{\tau}$ near $S_{2}$, where we write $A^{+}$for $A$ and $\phi_{k}^{+}$ for $\phi_{k}$. To study (14) in backward time near $S_{1}$ we apply $(\Delta, W) \rightarrow(-\Delta,-W)$ to $(46)$ to get

$$
\left\{\begin{array}{l}
\dot{Z}=-\left(A^{-}\left(W, B_{0}\right)+B_{2} \Delta \phi_{1}^{-}(\Delta, W, \tau)+B_{3} \Delta^{2} \phi_{2}^{-}(\Delta, W, \tau)\right. \\
\dot{\Delta}=-\frac{1}{2} \Delta W^{2} \\
\dot{W}=\frac{1}{2} W^{3},
\end{array}\right.
$$

with $A^{-}\left(W, B_{0}\right)=A^{+}\left(-W, B_{0}\right)$ and $\phi_{k}^{-}(\Delta, W, \tau)=(-1)^{k} \phi_{k}^{+}(-\Delta,-W, \tau), k=$ $1,2,3,4$. Hence for the study of (14) in backward time near $S_{1}$ we consider (47) on $\{\Delta \geq 0, W \geq 0, Z \leq 0\}$.

Proposition 3.6 implies the following relations:

$$
\left\{\begin{array}{l}
A^{-}\left(W, B_{0}\right)-A^{+}\left(W, B_{0}\right)=-\sqrt{2} B_{0} W\left(1+O\left(W^{2}\right)\right) \\
\phi_{2}^{-}(\Delta, W, \tau)-\phi_{2}^{+}(\Delta, W, \tau)=O\left(B_{0} W\right)+O\left(B_{2}\right) \\
\phi_{4}^{-}(\Delta, W, \tau)-\phi_{4}^{+}(\Delta, W, \tau)=O\left(B_{0} W\right)+O\left(B_{2}\right)+O(u) .
\end{array}\right.
$$

\subsubsection{Dulac maps $\mathcal{D}_{\tau}^{ \pm}$in normal coordinates $(Z, \Delta, W)$}

To study the transition near $S_{2}$, we work with (46) on the side $\{\Delta \geq 0, W \geq$ $0, Z \leq 0\}$. We define two sections $\Sigma_{+} \subset\left\{Z=-Z_{0}\right\}$ and $T_{+} \subset\left\{W=W_{0}\right\}$, for some small $Z_{0}>0, W_{0}>0$. Section $\Sigma_{+}$is parametrized by $(\delta, w) \in$ $\left[0, \delta_{0}\right] \times\left[0, w_{0}\right]$ for some $\delta_{0}, w_{0}>0$ and section $T_{+}$is parametrized by $(\Delta, Z) \in$ $\left[0, \Delta_{0}\right] \times\left[-Z^{\prime}, Z^{\prime}\right]$ for some $\Delta_{0}, Z^{\prime}>0$.

To study the transition map near the point $S_{1}$, we work with (47) on the side $\{\Delta \geq 0, W \geq 0, Z \leq 0\}$ and denote the transition map by $\mathcal{D}_{\tau}^{-}$between sections $\Sigma_{-} \subset\left\{Z=-Z_{0}\right\}$ and $T_{-} \subset\left\{W=W_{0}\right\}$, where $Z_{0}$ and $W_{0}$ are defined above. Parameterizations of $\Sigma_{-}$and $T_{-}$are also chosen in the same way.

The structure of $\mathcal{D}_{\tau}^{ \pm}$is given by:

Theorem 3.8. There are $C^{\infty}$-functions $\bar{A}^{ \pm}\left(w, w^{2} \ln w, B_{0}\right), \bar{\phi}_{k}^{ \pm}\left(\delta, w, w^{2} \ln w, \tau\right)$ of $\left(w, w^{2} \ln w, B_{0}\right),\left(\delta, w, w^{2} \ln w, \tau\right)$ such that $\mathcal{D}_{\tau}^{ \pm}:(\delta, w) \rightarrow(\Delta, Z)$ are given by $\mathcal{D}_{\tau}^{ \pm}(\delta, w)=\left(\frac{\delta w}{W_{0}}, Z_{\tau}^{ \pm}(\delta, w)\right)$ where

$$
Z_{\tau}^{ \pm}(\delta, w)=-Z_{0} \exp -\frac{1}{w^{2}}\left(\bar{A}^{ \pm}+B_{2} \delta \bar{\phi}_{1}^{ \pm}+B_{3} \delta^{2} \bar{\phi}_{2}^{ \pm}+u \delta^{3} \bar{\phi}_{3}^{ \pm}+\delta^{4} \bar{\phi}_{4}^{ \pm}\right)
$$

with $\bar{A}^{ \pm}\left(0,0, B_{0}\right)=1, \bar{\phi}_{1}^{ \pm}(0,0,0, \tau)= \pm 2 \sqrt{2} / 3, \bar{\phi}_{2}^{ \pm}(0,0,0, \tau)=1, \bar{\phi}_{3}^{ \pm}(0,0,0, \tau)=$ $\pm 4 \sqrt{2} / 5 \bar{H}(0, \lambda)$ and $\bar{\phi}_{4}^{ \pm}(0,0,0, \tau)=0$. Moreover,

$$
\begin{aligned}
& \bar{A}^{-}\left(w, w^{2} \ln w, B_{0}\right)-\bar{A}^{+}\left(w, w^{2} \ln w, B_{0}\right)=-2 \sqrt{2} B_{0} w(1+O(w)), \\
& \bar{\phi}_{2}^{-}\left(\delta, w, w^{2} \ln w, \tau\right)-\bar{\phi}_{2}^{+}\left(\delta, w, w^{2} \ln w, \tau\right)=O\left(B_{0} w\right)+O\left(B_{2}\right), \\
& \bar{\phi}_{4}^{-}\left(\delta, w, w^{2} \ln w, \tau\right)-\bar{\phi}_{4}^{+}\left(\delta, w, w^{2} \ln w, \tau\right)=O\left(B_{0} w\right)+O\left(B_{2}\right)+O(u) .
\end{aligned}
$$


Proof. We integrate the equation (46)/(47) from a point $\left(-Z_{0}, \delta, w\right) \in \Sigma_{ \pm}$to the point reached by the orbit on $T_{ \pm}$. Consider the trajectory $(Z(t), \delta(t), w(t))$ through $\left(-Z_{0}, \delta, w\right)$. Using the $(\Delta, W)$-component of (46) and (47) one finds

$$
(\delta(t), w(t))=\left(\delta\left(1-w^{2} t\right)^{\frac{1}{2}}, w\left(1-w^{2} t\right)^{-\frac{1}{2}}\right) .
$$

Clearly, we have $\delta(t) w(t)=\delta w=\epsilon$. This implies that the $\Delta$-component of $\mathcal{D}_{\tau}^{ \pm}$ is given by: $\Delta=\frac{\delta w}{W_{0}}$. The time we need to go from $\Sigma_{ \pm}$to $T_{ \pm}$is given by

$$
t(w)=\frac{1}{w^{2}}-\frac{1}{W_{0}^{2}}>0 .
$$

Integrating the $Z$-component of (46) and (47) we get

$$
\begin{aligned}
\int_{0}^{t} \frac{\dot{Z}(s)}{Z(s)} d s & =-\left(\int_{0}^{t} A^{ \pm}\left(w(s), B_{0}\right) d s+B_{2} \int_{0}^{t} \delta(s) \phi_{1}^{ \pm}(\delta(s), w(s), \tau) d s\right. \\
+ & B_{3} \int_{0}^{t} \delta(s)^{2} \phi_{2}^{ \pm}(\delta(s), w(s), \tau) d s+u \int_{0}^{t} \delta(s)^{3} \phi_{3}^{ \pm}(\delta(s), w(s), \tau) d s \\
& \left.+\int_{0}^{t} \delta(s)^{4} \phi_{4}^{ \pm}(\delta(s), w(s), \tau) d s\right) .
\end{aligned}
$$

Taking into account (50), (51), (52) and the initial condition $Z(0)=-Z_{0}$ we get the $Z$-component of $\mathcal{D}_{\tau}^{ \pm}$:

$$
\begin{aligned}
Z_{\tau}^{ \pm}(\delta, w)= & -Z_{0} \exp -\left(\int_{0}^{t(w)} A^{ \pm}\left(w(s), B_{0}\right) d s+B_{2} \delta \int_{0}^{t(w)}\left(1-w^{2} s\right)^{\frac{1}{2}} \phi_{1}^{ \pm} d s\right. \\
& +B_{3} \delta^{2} \int_{0}^{t(w)}\left(1-w^{2} s\right) \phi_{2}^{ \pm} d s+u \delta^{3} \int_{0}^{t(w)}\left(1-w^{2} s\right)^{\frac{3}{2}} \phi_{3}^{ \pm} d s \\
& \left.+\delta^{4} \int_{0}^{t(w)}\left(1-w^{2} s\right)^{2} \phi_{4}^{ \pm} d s\right)
\end{aligned}
$$

where $\phi_{k}^{ \pm}=\phi_{k}^{ \pm}(\delta(s), w(s), \tau)$, for $k=1,2,3$, 4 . If we use the change of variable: $\sigma=w^{2} s$ in (53), then we obtain:

$$
\int_{0}^{t(w)} A^{ \pm}\left(w(s), B_{0}\right) d s=\frac{1}{w^{2}} \int_{0}^{1-\frac{w^{2}}{W_{0}^{2}}} A^{ \pm}\left(w(1-\sigma)^{-\frac{1}{2}}, B_{0}\right) d \sigma
$$

and

$$
\begin{gathered}
\int_{0}^{t(w)}\left(1-w^{2} s\right)^{\frac{i}{2}} \phi_{i}^{ \pm}(\delta(s), w(s), \tau) d s \\
=\frac{1}{w^{2}} \int_{0}^{1-\frac{w^{2}}{W_{0}^{2}}}(1-\sigma)^{\frac{i}{2}} \phi_{i}^{ \pm}\left(\delta(1-\sigma)^{\frac{1}{2}}, w(1-\sigma)^{-\frac{1}{2}}, \tau\right) d \sigma, i=1,2,3,4 .
\end{gathered}
$$

To continue studying the integrals (54) and (55), we use the following proposition (see [DR09], Lemma 4.10. and Proposition 4.11.): 
Proposition 3.9. Let $r$ be $a C^{\infty}$-function and $i \geq 0$ an integer. One considers in the domain above defined for $(\delta, w, \tau)$ (in particular $0<w \leq w_{0}<W_{0}$ ) the integral function

$$
J(\delta, w, \tau)=\int_{0}^{1-\frac{w^{2}}{W_{0}^{2}}}(1-\sigma)^{\frac{i}{2}} r\left(\delta(1-\sigma)^{\frac{1}{2}}, w(1-\sigma)^{-\frac{1}{2}}, \tau\right) d \sigma .
$$

Then this function is equal to $s\left(\delta, w, w^{2} \ln w, \tau\right)$ where $s$ is a $C^{\infty}$-function. Moreover, one has that $J(0,0, \tau)=\frac{2}{i+2} r(0,0, \tau)$.

As a consequence of (54) and Proposition 3.9 (with $i=0$ ) one has that

$$
\int_{0}^{t(w)} A^{ \pm}\left(w(s), B_{0}\right) d s=\frac{1}{w^{2}} \bar{A}^{ \pm}\left(w, w^{2} \ln w, B_{0}\right)
$$

where $\bar{A}^{ \pm}$is a $C^{\infty}$-function in variable $\left(w, w^{2} \ln w, B_{0}\right)$ and $\bar{A}^{ \pm}\left(0,0, B_{0}\right)=$ $\frac{2}{0+2} A^{ \pm}\left(0, B_{0}\right)=1$. Using (55) we have, by Proposition 3.9 , that

$\int_{0}^{t(w)}\left(1-w^{2} s\right)^{\frac{i}{2}} \phi_{i}^{ \pm}(\delta(s), w(s), \tau) d s=\frac{1}{w^{2}} \bar{\phi}_{i}^{ \pm}\left(\delta, w, w^{2} \ln w, \tau\right), i=1,2,3,4$,

where $\bar{\phi}_{i}^{ \pm}$is a $C^{\infty}$-function of $\left(\delta, w, w^{2} \ln w, \tau\right)$ and $\bar{\phi}_{i}^{ \pm}(0,0,0, \tau)=\frac{2}{i+2} \phi_{i}^{ \pm}(0,0, \tau)$. Proposition 3.5 and the fact that $\phi_{i}^{-}(\delta, w, \tau)=(-1)^{i} \phi_{i}^{+}(-\delta,-w, \tau)$ imply that $\bar{\phi}_{1}^{ \pm}(0,0,0, \tau)= \pm \frac{2 \sqrt{2}}{3}, \bar{\phi}_{2}^{ \pm}(0,0,0, \tau)=1, \bar{\phi}_{3}^{ \pm}(0,0,0, \tau)= \pm \frac{4 \sqrt{2}}{5} \bar{H}(0, \lambda)$ and $\bar{\phi}_{4}^{ \pm}(0,0,0, \tau)=0$.

It remains to study $\bar{A}^{-}-\bar{A}^{+}, \bar{\phi}_{2}^{-}-\bar{\phi}_{2}^{+}$and $\bar{\phi}_{4}^{-}-\bar{\phi}_{4}^{+}$. Using the change of variable $s=w(1-\sigma)^{-\frac{1}{2}}$ and (48) we have that

$$
\begin{aligned}
\bar{A}^{-} & \left(w, w^{2} \ln w, B_{0}\right)-\bar{A}^{+}\left(w, w^{2} \ln w, B_{0}\right) \\
& =\int_{0}^{1-\frac{w^{2}}{W_{0}^{2}}}\left(A^{-}\left(w(1-\sigma)^{-\frac{1}{2}}, B_{0}\right)-A^{+}\left(w(1-\sigma)^{-\frac{1}{2}}, B_{0}\right)\right) d \sigma \\
& =2 w^{2} \int_{w}^{W_{0}} \frac{1}{s^{3}}\left(A^{-}\left(s, B_{0}\right)-A^{+}\left(s, B_{0}\right)\right) d s \\
& =-2 \sqrt{2} B_{0} w^{2} \int_{w}^{W_{0}} \frac{1}{s^{2}}\left(1+O\left(s^{2}\right)\right) d s \\
& =-2 \sqrt{2} B_{0} w^{2}\left(\frac{1}{w}-\frac{1}{W_{0}}+O(1)\right)=-2 \sqrt{2} B_{0} w(1+O(w)),
\end{aligned}
$$

where $O(w)$ is smooth in $\left(w, B_{0}\right)$. Similarly, we get

$$
\begin{aligned}
\bar{\phi}_{2}^{-}\left(\delta, w, w^{2} \ln w, \tau\right)-\bar{\phi}_{2}^{+}\left(\delta, w, w^{2} \ln w, \tau\right) \\
=\int_{0}^{1-\frac{w^{2}}{W_{0}^{2}}}(1-\sigma)\left(\phi_{2}^{-}-\phi_{2}^{+}\right)\left(\delta(1-\sigma)^{\frac{1}{2}}, w(1-\sigma)^{-\frac{1}{2}}, \tau\right) d \sigma \\
=B_{0} w \int_{0}^{1-\frac{w^{2}}{W_{0}^{2}}}(1-\sigma)^{\frac{1}{2}} f_{0}\left(\delta(1-\sigma)^{\frac{1}{2}}, w(1-\sigma)^{-\frac{1}{2}}, \tau\right) d \sigma \\
\quad+B_{2} \int_{0}^{1-\frac{w^{2}}{W_{0}^{2}}}(1-\sigma) f_{1}\left(\delta(1-\sigma)^{\frac{1}{2}}, w(1-\sigma)^{-\frac{1}{2}}, \tau\right) d \sigma \\
=B_{0} w F_{0}\left(\delta, w, w^{2} \ln w, \tau\right)+B_{2} F_{1}\left(\delta, w, w^{2} \ln w, \tau\right),
\end{aligned}
$$


where, by Proposition 3.9, $F_{0}$ and $F_{1}$ are smooth functions.

The study of $\bar{\phi}_{4}^{-}-\bar{\phi}_{4}^{+}$is analogous to the study of $\bar{\phi}_{2}^{-}-\bar{\phi}_{2}^{+}$.

Following Section 2.1.1, the vector field $-e^{-\widetilde{y}} Z_{\epsilon, \tau}^{(1)}$ is Hamiltonian, for $\epsilon=$ $B_{0}=0$. Since $T_{ \pm}$are visible in the family chart of the blow-up defined in Section 2.1, we can use $(h, \epsilon)$ as coordinates of $T_{ \pm}$, where $h$ is the value of the Hamiltonian $H(\widetilde{x}, \widetilde{y})=e^{-\widetilde{y}}\left(\widetilde{y}-\frac{1}{2} \widetilde{x}^{2}+1\right)$. We have $\epsilon=\Delta W_{0}$ and $h=h_{\tau, \epsilon}^{ \pm}(Z)$ where $(\Delta, Z)$ are the old coordinates of $T_{ \pm}$.

\section{Lemma 3.10.}

$$
\left\{\begin{aligned}
\epsilon=\Delta W_{0} & \\
h=h_{\tau, \epsilon}^{ \pm}(Z)= & \widetilde{h}_{\tau, \epsilon}(Z)+B_{0} \Pi_{0}^{ \pm}(Z, \tau, \epsilon)+B_{2} \epsilon \Pi_{1}^{ \pm}(Z, \tau, \epsilon) \\
& +u \epsilon^{3} \Pi_{3}^{ \pm}(Z, \tau, \epsilon)
\end{aligned}\right.
$$

where $\widetilde{h}_{\tau, \epsilon}, \Pi_{k}^{+}$are smooth in variable $(Z, \tau, \epsilon)$. The map $Z \rightarrow \widetilde{h}_{\tau, \epsilon}(Z)$ is a diffeomorphism, independent of \pm and has the form $\widetilde{h}_{\tau, \epsilon}(Z)=\epsilon \widetilde{\phi}(\tau, \epsilon)+\widetilde{h}^{1}(\tau, \epsilon) Z(1+$ $O(Z))$ with $\widetilde{h}^{1}(\tau, \epsilon)<0$.

Proof. See [Huz13].

From Theorem 3.8 and Lemma 3.10 follows the form of Dulac maps $\mathcal{D}_{\tau}^{ \pm}$from $\Sigma_{ \pm}$to $T_{ \pm}$, where $T_{ \pm}$is parametrized by $(h, \epsilon)$. We write $\mathcal{D}_{\tau}^{ \pm}(\delta, w)=\left(d_{\tau}^{ \pm}(\delta, w), \epsilon\right)$ where $\epsilon=\delta w$ and $d_{\tau}^{ \pm}(\delta, w)=h_{\tau, \epsilon}^{ \pm}\left(Z_{\tau}^{ \pm}(\delta, w)\right)$.

Theorem 3.11. There are $C^{\infty}$-functions $A^{ \pm}\left(\delta, w, w^{2} \ln w, \tau\right), \Phi_{k}^{ \pm}\left(\delta, w, w^{2} \ln w, \tau\right)$ of $\left(\delta, w, w^{2} \ln w, \tau\right)$ such that

$$
\left\{\begin{aligned}
d_{\tau}^{ \pm}(\delta, w)= & D^{ \pm}(\tau, \epsilon) \\
& +\exp -\frac{1}{w^{2}}\left(A^{ \pm}+B_{2} \delta \Phi_{1}^{ \pm}+u \delta^{3} \Phi_{3}^{ \pm}\right), \text {with } \\
D^{ \pm}(\tau, \epsilon)= & \epsilon \widetilde{\phi}(\tau, \epsilon)+B_{0} \Pi_{0}^{ \pm}(0, \tau, \epsilon)+B_{2} \epsilon \Pi_{1}^{ \pm}(0, \tau, \epsilon)+u \epsilon^{3} \Pi_{3}^{ \pm}(0, \tau, \epsilon) .
\end{aligned}\right.
$$

The functions $\Pi_{k}^{ \pm}, \widetilde{\phi}$ are introduced in Lemma 3.10, and $A^{ \pm}(0,0,0, \tau)=1$, $\Phi_{1}^{ \pm}(0,0,0, \tau)= \pm 2 \sqrt{2} / 3$ and $\Phi_{3}^{ \pm}(0,0,0, \tau)=( \pm 4 \sqrt{2} / 5) \bar{H}(0, \lambda)$. Moreover,

$$
A^{-}\left(\delta, w, w^{2} \ln w, \tau\right)-A^{+}\left(\delta, w, w^{2} \ln w, \tau\right)=-2 \sqrt{2} B_{0} w\left(1+O\left(\delta^{2}, w\right)\right) .
$$

Proof. See [Huz13].

\subsection{Global transition maps $\mathcal{F}_{\tau}, \mathcal{G}_{\tau}$}

In Section 3.1 we found an expression for the local Dulac maps $\mathcal{D}_{\tau}^{ \pm}: \Sigma_{ \pm} \rightarrow T_{ \pm}$ working with normal coordinates. It remains to study the regular transition maps $\mathcal{F}_{\tau}: \Sigma_{-} \rightarrow \Sigma_{+}$and $\mathcal{G}_{\tau}: T_{-} \rightarrow T_{+}$.

\subsubsection{Transition map $\mathcal{F}_{\tau}$}

We will state our main theorem in terms of the normal coordinates, as introduced in Section 3.1.5. To avoid confusion, let us parametrize $\Sigma_{-}=\left\{Z=-Z_{0}\right\}$ by normal coordinates $(\delta, w),(\delta, w) \sim(0,0), \delta \geq 0, w \geq 0$ and $\Sigma_{+}=\left\{Z=-Z_{0}\right\}$ by normal coordinates $(\Delta, W),(\Delta, W) \sim(0,0), \Delta \geq 0, W \geq 0$. Then: 
Theorem 3.12. The transition map $F_{\tau}:(\delta, w) \rightarrow(\Delta, W)$ has the following form:

$$
F_{\tau}(\delta, w)=\left(\delta\left(1+w^{2} R_{\tau}(\delta, w)\right), w\left(1+w^{2} R_{\tau}(\delta, w)\right)^{-1}\right),
$$

where $R_{\tau}=O\left(B_{0}, B_{2} \delta, u \delta^{3}\right)$ is a smooth family of functions in $(\delta, w, \tau)$.

Proof. See [Huz13].

\subsubsection{Transition map $\mathcal{G}_{\tau}$}

In Section 3.1.5 we have chosen two sections $T_{-}$and $T_{+}$with the same value $W_{0}: T_{-} \subset\left\{W=W_{0}\right\}$ and $T_{+} \subset\left\{W=W_{0}\right\}$, and we have parametrized them by $(h, \epsilon)$. We define a smooth transition map $\mathcal{G}_{\tau}(h, \epsilon)$ in the family directional chart by following the orbits of $-Z_{\epsilon, \tau}^{(1)}$, from $T_{-}$to $T_{+}$. (In the family chart, the blown up vector field $\bar{Z}_{\tau}$ is the $(\tau, \epsilon)$-family $Z_{\epsilon, \tau}^{(1)}$.) The map $\mathcal{G}_{\tau}$ has the following form

$$
\mathcal{G}_{\tau}(h, \epsilon)=(g(h, \epsilon, \tau), \epsilon) .
$$

Since $Z_{\epsilon, \tau}^{(1)}$ is invariant under $\mathcal{S}_{1}$, defined in (11), we have that orbits of $Z_{\epsilon, \tau}^{(1)}$ are symmetric with respect to the $\widetilde{y}$-axis if $B_{0}=B_{2}=u=0$. It is clear that level curves $\{H(\widetilde{x}, \widetilde{y})=h\}$ are also symmetric w.r.t. the $\widetilde{y}$-axis where the Hamiltonian $H$ is introduced in Section 2.1. Hence we obtain that

$$
g(h, \epsilon, \tau)=h \text { for } B_{0}=B_{2}=u=0 .
$$

Theorem 3.13. The h-component of the transition map $\mathcal{G}_{\tau}$ has the following form:

$$
g(h, \epsilon, \tau)=h+B_{0} l_{0}(h, \epsilon, \tau)+B_{2} \epsilon l_{1}(h, \epsilon, \tau)+u \epsilon^{3} l_{3}(h, \epsilon, \tau),
$$

for smooth functions $l_{0}, l_{1}, l_{3}$ such that $l_{0}(0,0, \tau)=-e \int_{-\widetilde{x}_{0}}^{\widetilde{x}_{0}} e^{-\frac{1}{2} \widetilde{x}^{2}} d \widetilde{x}+O\left(B_{0}\right)<0$ where $\widetilde{x}_{0}=\sqrt{2\left(1+W_{0}^{2}\right)} / W_{0}$.

Proof. See [Huz13].

\subsection{Difference map $\Omega_{\tau}$}

Let us recall the expression (18) of the difference map $\Omega_{\tau}$ :

$$
\Omega_{\tau}(\delta, w)=\mathcal{D}_{\tau}^{+} \circ \mathcal{F}_{\tau}(\delta, w)-\mathcal{G}_{\tau} \circ \mathcal{D}_{\tau}^{-}(\delta, w),
$$

where $\mathcal{D}_{\tau}^{ \pm}$is defined in Section 3.1, and $\mathcal{F}_{\tau}$ and $\mathcal{G}_{\tau}$ are defined in Section 3.2. Notice that we continue denoting the normalizing coordinates $(\Delta, W)$ near the point $S_{1}$ by $(\delta, w) . \Sigma_{-}$is parametrized by $(\delta, w)$.

Clearly the $\epsilon$-component of $\Omega_{\tau}(\delta, w)$ is equal to 0 . The $h$-component of $\Omega_{\tau}(\delta, w)$ is

$$
\omega_{\tau}(\delta, w)=d_{\tau}^{+}\left(\mathcal{F}_{\tau}(\delta, w)\right)-g\left(d_{\tau}^{-}(\delta, w), \delta w, \tau\right),
$$

where $d_{\tau}^{ \pm}$are the $h$-components of $\mathcal{D}_{\tau}^{ \pm}$. First we study each of the terms $g\left(d_{\tau}^{-}(\delta, w), \delta w, \tau\right)$ and $d_{\tau}^{+}\left(\mathcal{F}_{\tau}(\delta, w)\right)$. 


\subsubsection{The study of $d_{\tau}^{+} \circ \mathcal{F}_{\tau}$}

Combining Theorem 3.11 and Theorem 3.12 we obtain:

\section{Lemma 3.14.}

$$
\begin{aligned}
d_{\tau}^{+} \circ F_{\tau}(\delta, w)= & D^{+}(\tau, \epsilon)+\exp -\frac{1}{w^{2}}\left(\widetilde{A}^{+}\left(\delta, w, w^{2} \ln w, \tau\right)\right. \\
& \left.+B_{2} \delta \widetilde{\Phi}_{1}^{+}\left(\delta, w, w^{2} \ln w, \tau\right)+u \delta^{3} \widetilde{\Phi}_{3}^{+}\left(\delta, w, w^{2} \ln w, \tau\right)\right),
\end{aligned}
$$

where $\delta w=\epsilon, D^{+}(\tau, \epsilon)$ is defined in Theorem 3.11, $\widetilde{A}^{+}, \widetilde{\Phi}_{1}^{+}, \widetilde{\Phi}_{3}^{+}$are smooth functions in $\delta, w, w^{2} \ln w, \tau$ and

$$
\begin{gathered}
\widetilde{A}^{+}=A^{+}+O\left(w^{2} B_{0}\right), \\
\widetilde{\Phi}_{1}^{+}(0,0,0, \tau)=\Phi_{1}^{+}(0,0,0, \tau), \widetilde{\Phi}_{3}^{+}(0,0,0, \tau)=\Phi_{3}^{+}(0,0,0, \tau) .
\end{gathered}
$$

Proof. See [Huz13].

\subsubsection{The study of $g\left(d_{\tau}^{-}(\delta, w), \delta w, \tau\right)$}

Combining Theorem 3.11 and Theorem 3.13 we obtain:

Lemma 3.15. We have

$$
\begin{aligned}
g\left(d_{\tau}^{-}(\delta, w), \delta w, \tau\right)= & \bar{g}_{0}(\epsilon, \tau)+\exp -\frac{1}{w^{2}}\left(\widetilde{A}^{-}\left(\delta, w, w^{2} \ln w, \tau\right)\right. \\
& \left.+B_{2} \delta \widetilde{\Phi}_{1}^{-}\left(\delta, w, w^{2} \ln w, \tau\right)+u \delta^{3} \widetilde{\Phi}_{3}^{-}\left(\delta, w, w^{2} \ln w, \tau\right)\right),
\end{aligned}
$$

where $\delta w=\epsilon, \widetilde{A}^{-}, \widetilde{\Phi}_{1}^{-}, \widetilde{\Phi}_{3}^{-}$are smooth functions in $\delta, w, w^{2} \ln w, \tau$ and

$$
\bar{g}_{0}(\epsilon, \tau)=\widetilde{h}_{\tau, \epsilon}(0)+B_{0} \widetilde{\bar{l}}_{0}(\epsilon, \tau)+B_{2} \widetilde{\bar{l}}_{1}(\epsilon, \tau)+u \epsilon^{3} \widetilde{\bar{l}}_{3}(\epsilon, \tau),
$$

with $\widetilde{\bar{l}}_{j}$ smooth and $\widetilde{h}_{\tau, \epsilon}(0)$ introduced in Lemma 3.10. Moreover, $\widetilde{A}^{-}=A^{-}+$ $O\left(w^{2} B_{0}\right), \widetilde{\Phi}_{1}^{-}(0,0,0, \tau)=\Phi_{1}^{-}(0,0,0, \tau)$ and $\widetilde{\Phi}_{3}^{-}(0,0,0, \tau)=\Phi_{3}^{-}(0,0,0, \tau)$.

Proof. See [Huz13].

\subsubsection{Conclusion}

Combining Theorem 3.11, Lemma 3.14 and Lemma 3.15 we get

\section{Theorem 3.16.}

$$
\begin{aligned}
\omega_{\tau}(\delta, w)= & d_{\tau}^{+}\left(F_{\tau}(\delta, w)\right)-g\left(d_{\tau}^{-}(\delta, w), \delta w, \tau\right) \\
= & D^{+}(\tau, \epsilon)-\bar{g}_{0}(\epsilon, \tau)+\exp -\frac{1}{w^{2}}\left(\widetilde{A}^{+}+B_{2} \delta \widetilde{\Phi}_{1}^{+}+u \delta^{3} \widetilde{\Phi}_{3}^{+}\right) \\
& -\exp -\frac{1}{w^{2}}\left(\widetilde{A}^{-}+B_{2} \delta \widetilde{\Phi}_{1}^{-}+u \delta^{3} \widetilde{\Phi}_{3}^{-}\right),
\end{aligned}
$$

where $\bar{g}_{0}(\epsilon, \tau), D^{+}(\tau, \epsilon), \widetilde{A}^{ \pm}$and $\widetilde{\Phi}_{k}^{ \pm}$are introduced in Lemma 3.14 and Lemma 3.15. Moreover, we have

$$
\begin{gathered}
\widetilde{A}^{ \pm}(0,0,0, \tau)=1, \widetilde{\Phi}_{1}^{ \pm}(0,0,0, \tau)= \pm 2 \sqrt{2} / 3, \widetilde{\Phi}_{3}^{ \pm}(0,0,0, \tau)=( \pm 4 \sqrt{2} / 5) \bar{H}(0, \lambda) \\
\widetilde{A}^{-}\left(\delta, w, w^{2} \ln w, \tau\right)=\widetilde{A}^{+}\left(\delta, w, w^{2} \ln w, \tau\right)-2 \sqrt{2} B_{0} w\left(1+O\left(\delta^{2}, w\right)\right),
\end{gathered}
$$

and

$$
D^{+}(\tau, \epsilon)-\bar{g}_{0}(\epsilon, \tau)=B_{0} \kappa_{0}(\tau, \epsilon)+\epsilon B_{2} \kappa_{1}(\tau, \epsilon)+\epsilon^{3} u \kappa_{3}(\tau, \epsilon)
$$

where $\kappa_{j}$ is a smooth function and $\kappa_{0}$ is a strictly positive for $\left(\epsilon, B_{0}\right) \sim(0,0)$. 


\subsection{Lie-derivative}

Suppose that $(\epsilon, \tau)$ is arbitrary and fixed with $\epsilon \sim 0$ and $\epsilon>0$. (The case $\epsilon=0$ is trivial.) Our goal is to study the number of zeros of the function $\omega_{\tau}$ on each (1-dimensional) segment $s_{\epsilon}=\{(\delta, w) ; \delta w=\epsilon,(\delta, w) \in B(d), \delta \geq 0, w \geq 0\}$, where $B(d)$ is a ball of fixed radius $d>0$ at the origin $(\delta, w)=(0,0)$. We use the Lie-derivative $\mathcal{L}_{\mathcal{Y}} \omega_{\tau}=\delta \frac{\partial \omega_{\tau}}{\partial \delta}-w \frac{\partial \omega_{\tau}}{\partial w}$ of $\omega_{\tau}$ along the vector field $\mathcal{Y}=\delta \frac{\partial}{\partial \delta}-w \frac{\partial}{\partial w}$, which does not have singularities on $s_{\epsilon}$, and Rolle's theorem. As we will see in this section, instead of working with $\left\{\omega_{\tau}(\delta, w)=0\right\}$ it is more convenient to work with $\left\{\mathcal{L}_{\mathcal{Y}} \omega_{\tau}(\delta, w)=0\right\}$.

First, we prove some properties of the Lie-derivative $\mathcal{L}_{\mathcal{Y}}$ of functions which are smooth in $\left(\delta, w, w^{2} \ln w, \tau, \epsilon\right)$.

Lemma 3.17. If $n, m \in \mathbb{Z}$, then $\mathcal{L}_{\mathcal{Y}}\left(\delta^{n} w^{m}\right)=(n-m) \delta^{n} w^{m}$. If $F(\delta, w, \tau, \epsilon)=$ $f\left(\delta, w, w^{2} \ln w, \tau, \epsilon\right)$ with $f$ smooth, then $G(\delta, w, \tau, \epsilon)=\mathcal{L}_{\mathcal{Y}} F(\delta, w, \tau, \epsilon)$ is also smooth in $\left(\delta, w, w^{2} \ln w, \tau, \epsilon\right)$ and $G(0,0, \tau, \epsilon) \equiv 0$ (one can also write $G=o(1)$ ).

Proof. See [DR09], Lemma 5.9.

Lemma 3.17 implies the following lemma that will be useful later:

Lemma 3.18. Suppose that $n, m \in \mathbb{Z}$ and $\alpha \in \mathbb{R}$.

1. If $n \neq m$ and $F(\delta, w, \tau, \epsilon)=\delta^{n} w^{m}(\alpha+o(1))$ where o(1) is smooth in $\left(\delta, w, w^{2} \ln w, \tau, \epsilon\right)$ and $o(1) \equiv 0$ for $(\delta, w)=(0,0)$, then

$$
\mathcal{L}_{\mathcal{Y}} F(\delta, w, \tau, \epsilon)=(n-m) \delta^{n} w^{m}(\alpha+o(1)),
$$

where the symbol o(1) is also for a function smooth in $\left(\delta, w, w^{2} \ln w, \tau, \epsilon\right)$ and $o(1) \equiv 0$ for $(\delta, w)=(0,0)$.

2. If $n=m$, we have that

$$
\mathcal{L}_{\mathcal{Y}}\left(\delta^{n} w^{m}(\alpha+o(1))\right)=o\left(\delta^{n} w^{m}\right) .
$$

Proof. As a consequence of Lemma 3.17, we have that

$$
\begin{aligned}
\mathcal{L}_{\mathcal{Y}}\left(\delta^{n} w^{m}(\alpha+o(1))\right) & =(\alpha+o(1)) \mathcal{L}_{\mathcal{Y}}\left(\delta^{n} w^{m}\right)+\delta^{n} w^{m} \mathcal{L}_{\mathcal{Y}}(\alpha+o(1)) \\
& =(n-m) \delta^{n} w^{m}(\alpha+o(1))+\delta^{n} w^{m} o(1)
\end{aligned}
$$

As a consequence of Lemma 3.17, we have that

$$
\mathcal{L}_{\mathcal{Y}}\left(D^{+}(\tau, \epsilon)-\bar{g}_{0}(\epsilon, \tau)\right)=\mathcal{L}_{\mathcal{Y}}\left(D^{+}(\tau, \delta w)-\bar{g}_{0}(\delta w, \tau)\right)=0 .
$$

Thus, it suffices to study the Lie-derivative of the exponential terms in (65). If we write $T^{ \pm}(\delta, w, \tau, \epsilon)=\widetilde{A}^{ \pm}+B_{2} \delta \widetilde{\Phi}_{1}^{ \pm}+u \delta^{3} \widetilde{\Phi}_{3}^{ \pm}$, then we obtain

$$
\mathcal{L}_{\mathcal{Y}} \omega_{\tau}=-\mathcal{L}_{\mathcal{Y}}\left(\frac{1}{w^{2}} T^{+}\right) \exp -\frac{1}{w^{2}} T^{+}+\mathcal{L}_{\mathcal{Y}}\left(\frac{1}{w^{2}} T^{-}\right) \exp -\frac{1}{w^{2}} T^{-} .
$$

To push the terms $\mathcal{L} \mathcal{Y}\left(\frac{1}{w^{2}} T^{ \pm}\right)$in the corresponding exponential terms, we have to study $\mathcal{L}_{\mathcal{Y}}\left(\frac{1}{w^{2}} T^{ \pm}\right)$carefully. Using Lemma 3.17 we obtain that

$$
\begin{aligned}
\mathcal{L}_{\mathcal{Y}}\left(\frac{1}{w^{2}} T^{ \pm}\right) & =T^{ \pm} \mathcal{L}_{\mathcal{Y}}\left(\frac{1}{w^{2}}\right)+\frac{1}{w^{2}} \mathcal{L}_{\mathcal{Y}}\left(T^{ \pm}\right) \\
& =2 \frac{1}{w^{2}} T^{ \pm}+\frac{1}{w^{2}} \mathcal{L}_{\mathcal{Y}}\left(T^{ \pm}\right)=\frac{1}{w^{2}}\left(2 T^{ \pm}+\mathcal{L}_{\mathcal{Y}}\left(T^{ \pm}\right)\right) .
\end{aligned}
$$


Let us remind that $T^{ \pm}$is smooth in $\left(\delta, w, w^{2} \ln w, \tau, \epsilon\right)$. Then, by Lemma 3.17, we get

$$
2 T^{ \pm}(0,0, \tau, \epsilon)+\mathcal{L}_{\mathcal{Y}}\left(T^{ \pm}\right)(0,0, \tau, \epsilon)=2 \widetilde{A}^{ \pm}(0,0,0, \tau)+0=2>0 .
$$

It follows that the function $P^{ \pm}(\delta, w, \tau, \epsilon):=2 T^{ \pm}(\delta, w, \tau, \epsilon)+\mathcal{L}_{Y}\left(T^{ \pm}\right)(\delta, w, \tau, \epsilon)$ is strictly positive by taking a sufficiently small ball $B(d)$ at the origin $(\delta, w)=$ $(0,0)$. Since $P^{ \pm}$is smooth in $\left(\delta, w, w^{2} \ln w, \tau, \epsilon\right)$, its logarithm is also a smooth function in $\left(\delta, w, w^{2} \ln w, \tau, \epsilon\right)$. So, we can write

$$
\begin{aligned}
\mathcal{L}_{\mathcal{Y}} \omega_{\tau} & =-\frac{1}{w^{2}} P^{+} \exp -\frac{1}{w^{2}} T^{+}+\frac{1}{w^{2}} P^{-} \exp -\frac{1}{w^{2}} T^{-} \\
& =-\frac{1}{w^{2}}\left(\exp -\frac{1}{w^{2}}\left(T^{+}-w^{2} \ln P^{+}\right)-\exp -\frac{1}{w^{2}}\left(T^{-}-w^{2} \ln P^{-}\right)\right) .
\end{aligned}
$$

The expression (66) implies that the equation $\left\{\mathcal{L}_{\mathcal{Y}} \omega_{\tau}=0\right\}$ is equivalent to

$$
T^{+}-T^{-}+w^{2} \ln \frac{P^{-}}{P^{+}}=0,
$$

for $w>0$. Using the properties of $\widetilde{A}^{ \pm}, \widetilde{\Phi}_{1}^{ \pm}$and $\widetilde{\Phi}_{3}^{ \pm}$given in Theorem 3.16 we have that

$$
\begin{gathered}
T^{+}-T^{-}=\widetilde{A}^{+}-\widetilde{A}^{-}+B_{2} \delta\left(\widetilde{\Phi}_{1}^{+}-\widetilde{\Phi}_{1}^{-}\right)+u \delta^{3}\left(\widetilde{\Phi}_{3}^{+}-\widetilde{\Phi}_{3}^{-}\right) \\
=2 \sqrt{2} B_{0} w\left(1+O\left(\delta^{2}, w\right)\right)+B_{2} \delta \frac{4 \sqrt{2}}{3}\left(1+o_{1}(1)\right)+u \delta^{3} \frac{8 \sqrt{2}}{5}\left(\bar{H}(0, \lambda)+o_{3}(1)\right),
\end{gathered}
$$

where $O\left(\delta^{2}, w\right), o_{1}(1)$ and $o_{3}(1)$ are smooth in $\left(\delta, w, w^{2} \ln w, \tau\right)$.

Let us study $w^{2} \ln \frac{P^{-}}{P^{+}}$. We have

$$
P^{-}-P^{+}=2\left(T^{-}-T^{+}\right)+\mathcal{L}_{\mathcal{Y}}\left(T^{-}-T^{+}\right) .
$$

Keeping in mind Lemma 3.18 and (68) we obtain that

$$
\begin{aligned}
& \mathcal{L}_{\mathcal{Y}}\left(T^{-}-T^{+}\right)=-2 \sqrt{2} B_{0} \mathcal{L}_{\mathcal{Y}}\left(w\left(1+O\left(\delta^{2}, w\right)\right)\right) \\
&-B_{2} \frac{4 \sqrt{2}}{3} \mathcal{L}_{\mathcal{Y}}\left(\delta\left(1+o_{1}(1)\right)\right)-u \frac{8 \sqrt{2}}{5} \mathcal{L}_{\mathcal{Y}}\left(\delta^{3}\left(\bar{H}(0, \lambda)+o_{3}(1)\right)\right) \\
&=2 \sqrt{2} B_{0} w\left(1+O\left(\delta^{2}, w\right)\right)-B_{2} \frac{4 \sqrt{2}}{3} \delta\left(1+o_{1}(1)\right)-u \frac{24 \sqrt{2}}{5} \delta^{3}\left(\bar{H}(0, \lambda)+o_{3}(1)\right),
\end{aligned}
$$

for some new functions $O\left(\delta^{2}, w\right), o_{1}(1), o_{3}(1)$ smooth in $\left(\delta, w, w^{2} \ln w, \tau\right)$. This implies that

$$
\begin{gathered}
P^{-}=P^{+}+2\left(T^{-}-T^{+}\right)+\mathcal{L}_{Y}\left(T^{-}-T^{+}\right) \\
=P^{+}-2 \sqrt{2} B_{0} w\left(1+O\left(\delta^{2}, w\right)\right)-B_{2} 4 \sqrt{2} \delta\left(1+o_{1}(1)\right)-u 8 \sqrt{2} \delta^{3}\left(\bar{H}(0, \lambda)+o_{3}(1)\right),
\end{gathered}
$$

for some new functions $O\left(\delta^{2}, w\right), o_{1}(1), o_{3}(1)$.

From the above expression and the fact that $P^{-}(0,0, \tau, \epsilon)=2>0$ it follows that

$$
\frac{P^{-}}{P^{+}}=1-\sqrt{2} B_{0} w(1+o(1))-B_{2} 2 \sqrt{2} \delta\left(1+o_{1}(1)\right)-u 4 \sqrt{2} \delta^{3}\left(\bar{H}(0, \lambda)+o_{3}(1)\right),
$$


for some functions $o(1), o_{1}(1), o_{3}(1)$ smooth in $\left(\delta, w, w^{2} \ln w, \tau\right)$. Finally, we have

$$
w^{2} \ln \frac{P^{-}}{P^{+}}=B_{0} w O\left(w^{2}\right)+B_{2} \delta O_{1}\left(w^{2}\right)+u \delta^{3} O_{3}\left(w^{2}\right),
$$

where $O\left(w^{2}\right), O_{1}\left(w^{2}\right)$ and $O_{3}\left(w^{2}\right)$ are smooth in $\left(\delta, w, w^{2} \ln w, \tau\right)$.

If we use now the expressions (68) and (69) we obtain that

$$
\begin{gathered}
T^{+}-T^{-}+w^{2} \ln \frac{P^{-}}{P^{+}}= \\
2 \sqrt{2} B_{0} w\left(1+O\left(\delta^{2}, w\right)\right)+B_{2} \delta \frac{4 \sqrt{2}}{3}\left(1+o_{1}(1)\right)+u \delta^{3} \frac{8 \sqrt{2}}{5}\left(\bar{H}(0, \lambda)+o_{3}(1)\right),
\end{gathered}
$$

where $O\left(\delta, w^{2}\right), o_{1}(1)$ and $o_{3}(1)$ are smooth in $\left(\delta, w, w^{2} \ln w, \tau\right)$. Thus, the equation $\left\{\mathcal{L}_{\mathcal{Y}} w_{\tau}=0\right\}$ is equivalent, for $w>0$, to:

$$
\begin{aligned}
2 \sqrt{2} B_{0} w\left(1+O\left(\delta^{2}, w\right)\right)+ & B_{2} \delta \frac{4 \sqrt{2}}{3}\left(1+o_{1}(1)\right) \\
& +u \delta^{3} \frac{8 \sqrt{2}}{5}\left(\bar{H}(0, \lambda)+o_{3}(1)\right)=0 .
\end{aligned}
$$

\subsection{A finite cyclicity of $L_{0}$}

As mentioned above, we distinguish between the case $B_{2} \neq 0$ and the case $B_{2} \sim 0$ and $\bar{H}(0, \lambda) \neq 0$ for all $\lambda \in \Lambda$.

\subsection{1 $\left.B_{2} \in C \subset\right]-\infty,+\infty[\backslash\{0\}, C$ is a compact set in $\mathbb{R}$}

We can write (70) as

$$
2 \sqrt{2} B_{0} w\left(1+O\left(\delta^{2}, w\right)\right)+B_{2} \delta \frac{4 \sqrt{2}}{3}(1+o(1))=0,
$$

where $O\left(\delta^{2}, w\right)$ and $o(1)$ are smooth in $\left(\delta, w, w^{2} \ln w, \tau\right)$. To prove Theorem 2.1 (i), we use an algorithm of derivation-division introduced in [DR09].

We begin the derivation-division algorithm by dividing (71) by $2 \sqrt{2} w(1+$ $O\left(\delta^{2}, w\right)$ ), the factor of the parameter $B_{0}$, which is strictly positive on segments $s_{\epsilon}, \epsilon>0$. Then the left-hand side of (71) changes to

$$
Q:=B_{0}+B_{2} \delta w^{-1} \frac{2}{3}(1+o(1)),
$$

for a new $o(1)$ smooth in $\left(\delta, w, w^{2} \ln w, \tau\right)$. By Lemma 3.18, we get

$$
\mathcal{L}_{\mathcal{Y}}(Q)=B_{2} \delta w^{-1} \frac{4}{3}(1+o(1))
$$

where $o(1)$ is different from the previous one. As $B_{2} \neq 0$, this function is nonzero for $(\delta, w) \sim(0,0), \delta>0, w>0, B_{0} \sim 0, B_{2} \in C, B_{3} \in \mathcal{B}, u \sim 0$ and $\lambda \in \Lambda$. Following Rolle's Theorem, at most 2 limit cycles occur Hausdorff close to $L_{0}$, for $B_{2} \neq 0$. 


\subsection{2 $B_{2} \sim 0$ and $\bar{H}(0, \lambda) \neq 0$ for all $\lambda \in \Lambda$}

We again use an algorithm of derivation-division. We introduce $\left(\bar{B}_{0}, \bar{B}_{2}, \bar{u}\right)$, where $\left(\bar{B}_{0}, \bar{B}_{2}, \bar{u}\right) \in \mathbb{S}^{2}$ and

$$
\left(B_{0}, B_{2}, u\right)=r\left(\bar{B}_{0}, \bar{B}_{2}, \bar{u}\right)
$$

where $r \geq 0, r \sim 0$. For $r>0$, using this rescaling, the expression (70) can be written as

$$
\begin{aligned}
\bar{Q}\left(\delta, w, r, \bar{B}_{0}, \bar{B}_{2}, \bar{u}, B_{3}, \lambda\right):= & 2 \sqrt{2} \bar{B}_{0} w\left(1+O\left(\delta^{2}, w\right)\right)+\bar{B}_{2} \delta \frac{4 \sqrt{2}}{3}\left(1+o_{1}(1)\right) \\
& +\bar{u} \delta^{3} \frac{8 \sqrt{2}}{5}\left(\bar{H}(0, \lambda)+o_{3}(1)\right)=0 .
\end{aligned}
$$

(If $r=0$, then the left-hand side of (70) is equal to zero, corresponding to a system of center type.) Instead of using coordinates on the sphere, it is more convenient to use different charts of the sphere.

(1) Case $\bar{u}= \pm 1,\left(\bar{B}_{0}, \bar{B}_{2}\right) \in K, K$ is any compact subset of $\mathbb{R}^{2}$. We begin the derivation-division algorithm by dividing (74) by

$$
T_{1}=2 \sqrt{2} w\left(1+O\left(\delta^{2}, w\right)\right),
$$

the factor of the parameter $\bar{B}_{0}$ in (74), which is strictly positive on segments $s_{\epsilon}$, for each $\epsilon \sim 0$ and $\epsilon>0$. Using Lemma 3.18 we obtain that

$$
\mathcal{L}_{\mathcal{Y}}\left(\frac{\bar{Q}}{T_{1}}\right)=\bar{B}_{2} \delta w^{-1} k_{1}\left(1+o_{1}(1)\right)+\bar{u} \delta^{3} w^{-1} k_{3}\left(\bar{H}(0, \lambda)+o_{3}(1)\right),
$$

for some positive constant $k_{1}$ and $k_{3}$ and some new $o_{1}(1)$ and $o_{3}(1)$. Hence, we have eliminated the parameter $\bar{B}_{0}$. Let us divide now (75) by the positive factor $T_{2}=\delta w^{-1} k_{1}\left(1+o_{1}(1)\right)$ of $\bar{B}_{2}$ in (75). Then, by Lemma 3.18 ,

$$
\mathcal{L}_{\mathcal{Y}}\left(\frac{\mathcal{L}_{\mathcal{Y}}\left(\frac{\bar{Q}}{T_{1}}\right)}{T_{2}}\right)=\bar{u} \delta^{2} \bar{k}_{3}\left(\bar{H}(0, \lambda)+o_{3}(1)\right)
$$

where $\bar{k}_{3}$ is a positive constant and $o_{3}(1)$ is smooth in $\left(\delta, w, w^{2} \ln w, \tau\right)$.

As $\bar{u}= \pm 1$ and $\bar{H}(0, \lambda) \neq 0$ for all $\lambda \in \Lambda$, the expression (76) is nonzero, for $(\delta, w) \sim(0,0),(\delta, w)>(0,0), r \sim 0,\left(\bar{B}_{0}, \bar{B}_{2}\right) \in K, B_{3} \in \mathcal{B}$ and $\lambda \in \Lambda$. Now Rolle's Theorem implies that $\bar{Q}$ has at most 2 roots (counting multiplicity) on the segments $s_{\epsilon}$ under the given conditions on the parameters.

Remark 2. Since $\bar{H}(0, \lambda) \neq 0$ for all $\lambda \in \Lambda$, in each step of the above algorithm of derivation-division we deal with factors $\delta^{n} w^{m}(\alpha+o(1))$, where $\alpha \neq 0$ and $n, m \in \mathbb{Z}, n \neq m$. This enables us to have a well-defined algorithm in each step.

(2) Case $\bar{B}_{0}= \pm 1,\left(\bar{B}_{2}, \bar{u}\right) \in K, K$ is any compact set, and (3) Case $\bar{B}_{2}= \pm 1$, $\left(\bar{B}_{0}, \bar{u}\right) \in K, K$ is any compact set. The study of the case (2) and the case (3) is analogous to the study of the case (1); we can prove that $\bar{Q}$ has at most 2 roots (counting multiplicity) on the segments $s_{\epsilon}$.

As the equation (70) is equivalent to $\left\{\mathcal{L}_{\mathcal{Y}} \omega_{\tau}=0\right\}$ for $w>0$, the Rolle's theorem implies that the cyclicity of $L_{0}$ at $B_{2}=0$ is bounded by 3 under the given condition on the function $\bar{H}$. Hence we arrive at the statement of Theorem 2.1 (ii). 


\section{References}

[Ben81] É. Benoît. Chasse au canard. II. Tunnels - entonnoirs-peignes. Collect. Math., 32(2):77-97, 1981.

[DMDR11] P. De Maesschalck, F. Dumortier, and R. Roussarie. Cyclicity of common slow-fast cycles. Indag. Math. (N.S.), 22(3-4):165-206, 2011.

[DR96] F. Dumortier and R. Roussarie. Canard cycles and center manifolds. Mem. Amer. Math. Soc., 121(577):x+100, 1996. With an appendix by Cheng Zhi Li.

[DR09] F. Dumortier and R. Roussarie. Birth of canard cycles. Discrete Contin. Dyn. Syst. Ser. S, 2(4):723-781, 2009.

[DR10] F. Dumortier and R. Roussarie. Smooth normal linearization of vector fields near lines of singularities. Qual. Theory Dyn. Syst., 9(1-2):39-87, 2010.

[HDMD13] R. Huzak, P. De Maesschalck, and F. Dumortier. Limit cycles in slow-fast codimension 3 saddle and elliptic bifurcations. J. Differential Equations, 255(11):4012-4051, 2013.

[HDMD14] R. Huzak, P. De Maesschalck, and F. Dumortier. Primary birth of canard cycles in slow-fast codimension 3 elliptic bifurcations. Communications on Pure and Applied Analysis, 13(6):2641-2673, 2014.

[Huz13] R. Huzak. Limit cycles in slow-fast codimension 3 bifurcations. PhD thesis, Hasselt University, Belgium, 2013.

[Huz16] R. Huzak. Cyclicity of the origin in slow-fast codimension 3 saddle and elliptic bifurcations. Discrete Contin. Dyn. Syst., 36(1):171$215,2016$.

[Huz17] R. Huzak. Normal forms of Liénard type for analytic unfoldings of nilpotent singularities. Proc. Amer. Math. Soc., 145(10):4325-4336, 2017.

[KS01] M. Krupa and P. Szmolyan. Relaxation oscillation and canard explosion. J. Differential Equations, 174(2):312-368, 2001. 